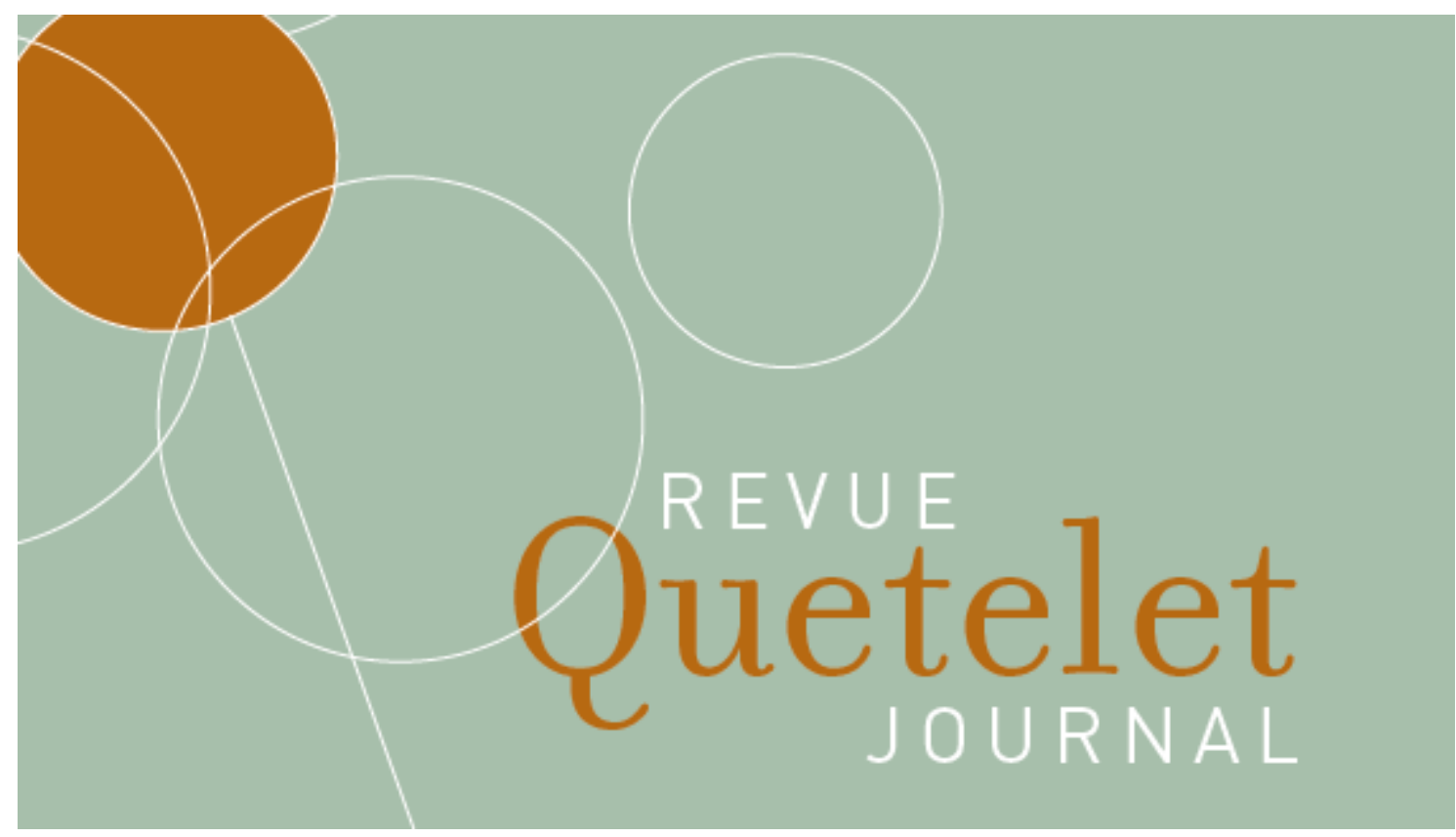

Vol. 8, $\mathrm{n}^{\circ} 1,2020$, pp. 37-72

DOI : $10.14428 /$ rqj2020.08.01.02

ISSN: 2593-9157

\title{
Les causes de décès aux grands âges en France, évolution récente
}

France Meslé, Jacques Vallin

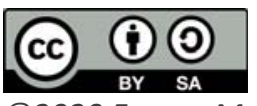

(C)2020 France Meslé, Jacques Vallin

This work is licensed under a Creative Commons Attribution-NonCommercial 4.0 International License. You can share, adapt the material for non-commercial purposes provided that you give appropriate credit and indicate if changes were made. For details see https://creativecommons.org/licenses/by-sa/4.0/

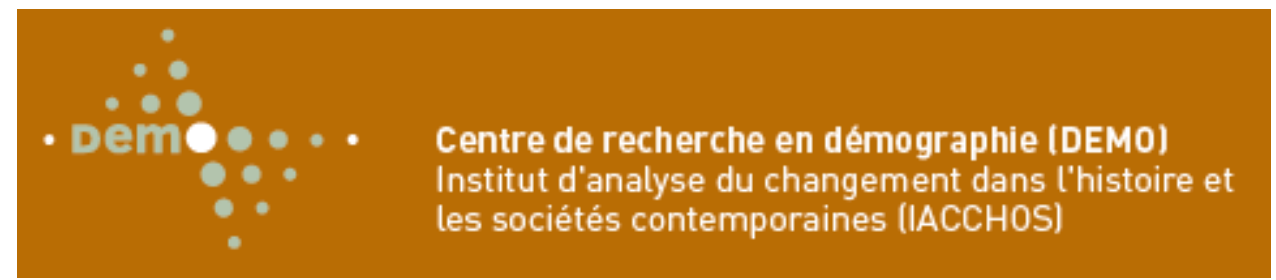





\title{
Les causes de décès aux grands âges en France, évolution récente
}

\author{
France MesLé \\ JACQUES VALLIN ${ }^{1}$
}

\begin{abstract}
Résumé
Pendant longtemps il a été jugé vain de s'intéresser aux causes de décès aux grands âges, tant il semblait difficile de poser un diagnostic fiable sur les événements ayant concouru à la mort d'un individu dont l'âge lui-même rendait la fin inévitable. Un âge que d'ailleurs fort peu de privilégiés pouvaient atteindre. Le flou des causes déclarées se dissipait alors dans le puits sans fond d'un vaste groupe d'âges terminal de 85 ans et plus, voire 80 ans et plus ! À une époque où l'espérance de vie dépasse 80 ans, et même 85 ans pour les femmes, cette posture n'est plus tenable. Si l'on veut connaitre et comprendre les ressorts du progrès à venir de la longévité humaine, il faut briser le tabou de la qualité des données et s'efforcer d'apprendre de quoi l'on meurt aux plus grands âges.

Grâce aux données aujourd'hui très détaillées fournies par l'INSERM, il a été possible d'étudier le poids des différentes causes de décès dans l'évolution de l'espérance de vie à 90 ans de 1979 à 2017. Cette étude révèle, entre autres, trois aspects importants de la mortalité des personnes très âgées.

Le premier, en apparence anecdotique, les effets de la canicule de 2003, livre un enseignement plus général que ce que l'on peut dire des conséquences directes de cette vague de chaleur. Celle-ci a en effet révélé qu'une plus grande attention au quotidien des personnes très âgées pouvait améliorer sensiblement leur survie en réduisant certaines causes de décès qui ne sont pas forcément celles qu'elle avait le plus amplifiées («démences et maladies neurodégénératives», «maladies du cœur»), puisqu'en 2004 on a observé un recul important des «maladies infectieuses et respiratoires», des "autres maladies de l'appareil circulatoire», des «autres maladies» des «morts violentes» et de la «sénilité», beaucoup moins affectées en négatif par la canicule.

Le second, plus général, fait la lumière sur les groupes de causes de décès dont le recul a fait progresser l'espérance de vie à 90 ans au cours des 35 dernières années. Le rôle principal a été joué par le succès spectaculaire de la lutte contre les maladies
\end{abstract}

1. Institut National d'Études Démographiques (INED), Paris, France. 
cérébro-vasculaires. Mais le recul des «maladies du cœur» ainsi que celui des «maladies infectieuses et respiratoires» y ont aussi beaucoup contribué. Quant au retournement de la mortalité par "tumeurs» chez les hommes, il explique celui de la surmortalité masculine elle-même. À l'inverse, la montée du groupe des démences et autres maladies neuro-dégénératives est réelle même si elle doit être interprétée avec prudence en raison des confusions entre ce groupe et la «sénilité» qui, encore forte dans les années 1980 a beaucoup diminué, biaisant un peu les tendances observées. Le troisième n'est pas le moindre : la qualité des données a beaucoup progressé. Pour preuve, la "sénilité», longtemps utilisée comme fourre-tout de causes mal diagnostiquées, a régressé au point qu'elle pourrait fort bien aujourd'hui devenir une vraie "cause initiale» reflétant une situation où l'usure générale de l'organisme crée une fragilité indépendante de telle ou telle cause particulière.

\section{Mots-clé}

Personnes âgées, espérance de vie, mortalité, cause de décès, maladies cérébro-vasculaires, maladies de cœur, maladies infectieuses et respiratoires, sénilité, qualité des données, canicule, surmortalité masculine.

\footnotetext{
Abstract

For many years it was considered pointless to study causes of death at advanced ages, given the difficulty of making a reliable diagnosis based on the possible contributors to the death of an individual whose age was reaching its natural limit. For the vast terminal age group, defined as ages $85+$ or even $80+$, the reported causes of death were vague and inconsistent to say the least. Now that expectation of life exceeds 80 years, and even 85 years for women, such an approach is no longer acceptable. If we want to identify and understand the drivers of future progress in human longevity, we need to break the taboo of data quality and seek to identify the main causes of death among the oldest-old.

Using detailed data made available by INSERM, it is now possible to measure the contribution of changes in various causes of death to the improvement (or deterioration) of life expectancy at age 90, from 1979 to 2017. This study reveals, inter alia, three important aspects of oldest-old mortality.

The first somewhat anecdotal aspect, linked to the effects of the 2003 heatwave, provides more general insights than the lessons drawn from the immediate consequences of the event itself. The data reveal that greater attention to the daily needs of elderly people led to unexpected progress in their survival by reducing certain causes of death which were not necessarily those amplified by the heatwave (dementia and neurodegenerative diseases, heart diseases). This is evidenced by the substantial decrease in 2004 in deaths from infectious and respiratory diseases, other circulatory diseases, other diseases, deaths from external causes and senility, which were much less impacted by the heatwave.

Secondly, and more generally, the study sheds light on the main groups of causes whose decline has contributed to the increase in life expectancy at age 90 over the last 35 years. Spectacular success in the fight against cerebrovascular diseases has been the main driver of progress. But the decline in heart diseases and in infectious
} 
and respiratory diseases has also made a strong contribution. At the same time, the trend reversal in neoplasms among men is a key factor behind the similar pattern of decrease in overall male excess mortality. Conversely, the sharp increase in dementia and neurodegenerative diseases appears to be real, even if significantly exaggerated due to the confusion of this group with that of "senility", still commonly reported as a cause of death in the 1980s but much less so since then.

Thirdly, but no less decisively, data quality has improved substantially. This is illustrated by the fact that "senility», a category long used as a catch-all for poorly diagnosed causes, has declined so sharply that it may well be redeployed as a "fundamental cause", reflecting a specific health status where general wear and tear of the human body results in a frailty that cannot be imputed to any specific cause.

\section{Keywords}

Elderly, life expectancy, mortality, cause of death, cerebrovascular diseases, heart diseases, infectious and respiratory diseases, senility, data quality, heatwave, male excess mortality.

L'analyse des causes médicales de décès est un instrument précieux pour interpréter les évolutions de la mortalité ou comprendre ce qui la différencie d'une population à l'autre. On a longtemps considéré, cependant que, passé un certain âge, déterminer la cause de décès est de plus en plus vain (Gittelsohn, Senning, 1979 ; Myers, Manton, 1983 ; Meslé, 2006), car de nombreux processus morbides peuvent s'entremêler et priver de sens la sélection d'une cause dite principale ou initiale comme étant la cause réellement responsable du décès, sans parler du fait qu'aux grands âges la dégradation générale de l'organisme et de ses ressorts vitaux peut paraître l'emporter sur toute maladie ou traumatisme particulier. Il n'y pas si longtemps encore, d'un bout à l'autre de la chaîne d'observation, il y avait une sorte de consensus pour ne pas trop en faire sur le grand âge : le médecin certificateur se contentant de déclarer des causes très floues tandis que le statisticien bloquait sa distribution par âge des causes de décès par un groupe d'âges ouvert, aussi large que vague, à 80 ans et plus ou 85 ans et plus, jetant ainsi un voile pudique sur la qualité réputée mauvaise des causes de décès déclarées chez les vieillards. Aux plus grands âges il y a eu aussi pendant longtemps un certain flou sur la qualité des âges déclarés, même si de grands progrès ont été faits à la fin du siècle dernier dans les pays développés (Kannisto, 1994).

Ce n'est guère que depuis les années 1990, notamment avec la mise en usage de la 10ème révision de la Classification internationale des maladies (CIM), que l'OMS s'efforce de plus en plus d'obtenir des instituts nationaux des données par âge jusqu'à 95 ans. 
Aujourd'hui encore, bien que de telles données soient de plus en plus souvent accessibles, elles restent, au-delà d'un certain âge, relativement peu utilisées parce que toujours suspectées d'être de mauvaise qualité ou, au mieux, trop difficiles à interpréter. Il est pourtant de plus en plus important de s'intéresser à la mortalité aux grands âges. En France, par exemple, la moitié des décès féminins observés en 2016 ont emporté des femmes de plus de 86 ans et $35 \%$ sont survenus à plus de 90 ans. En 1950, c'était $92 \%$ des décès qui se produisaient avant 86 ans contre $8 \%$ seulement après (et même à peine $2 \%$ après 90 ans). De ce point de vue, les temps ont complètement changé. Certes savoir de quoi meurent les femmes de moins de 86 ans reste un enjeu de santé publique majeur et l'on pourrait même dire que c'est d'autant plus important que cela devient presque rare. Mais c'est un enjeu démographique et social encore plus grand de savoir de quoi elles meurent après 86 ans ou même après 90 ans dans la mesure où elles sont de plus en plus nombreuses à vivre au-delà de ces âges et que ce phénomène risque fort de s'amplifier à l'avenir.

Il est d'autant plus important de savoir de quoi meurent les personnes très âgées que la crise provoquée par la canicule de 2003 a mis en évidence, qu'à condition d'y prêter attention, il est des aspects de la survie aux grands âges que l'on peut très nettement améliorer. La forte alerte donnée par le surcroît de décès dus à la vague de chaleur a poussé les pouvoirs publics et les acteurs de santé à améliorer leurs pratiques de soins quotidiens aux personnes âgées et cela n'a pas eu seulement pour effet d'atténuer la crise et de rattraper très vite la petite chute d'espérance de vie qu'avait provoquée la canicule (Hémon, Jougla, 2004), mais aussi de retrouver l'année suivante une espérance de vie nettement plus haute que n'aurait donné la simple poursuite de la tendance antérieure à la crise (Rey et al., 2007 ; Toulemon, Barbieri, 2008). Indubitablement, il existe aux grands âges des causes de décès sur lesquelles il serait sûrement utile d'agir. Ce n'est évidemment pas là le seul aspect ni sans doute le plus important de l'évolution récente des causes de décès aux grands âges, mais c'est un fait qui devrait au moins pousser notre curiosité à en savoir plus sur le sujet. Quelles sont ces causes ? Peut-on les mesurer? Comment évoluent-elles ? Nous allons ici porter toute notre attention à la mortalité après 90 ans, notamment chez les femmes qui ont été jusqu'ici à l'avant-garde du progrès.

Après quelques précisions sur la canicule et ses conséquences, nous analyserons plus largement comment ont évolué les principales causes de décès au cours des dernières décennies en examinant les ruptures et les continuités. Nous nous pencherons ensuite sur un phénomène majeur qui 
caractérise la période : le renversement de tendance de la différence entre femmes et hommes. Et, pour terminer, nous reviendrons sur la question de la qualité des données.

\section{Données et méthodes}

Nous partons ici d'enregistrements individuels anonymisés de décès fournis annuellement par l'Institut national de la santé et de la recherche médicale (INSERM). Ces enregistrements varient dans le temps, d'une part en raison de la nomenclature de causes de décès utilisée mais aussi en fonction de la manière dont l'âge est identifié. Pour minimiser le problème posé par les changements de nomenclature, nous nous limitons ici à l'étude des années 1979-1999 régies par la 9ème révision de la Classification internationale des maladies (CIM-9) et des années 2000-2015 régies par la 10ème révision de la CIM (CIM-10).

FIgURE 1 Diagramme de Lexis représentant les données par âge utilisées

Année de naissance

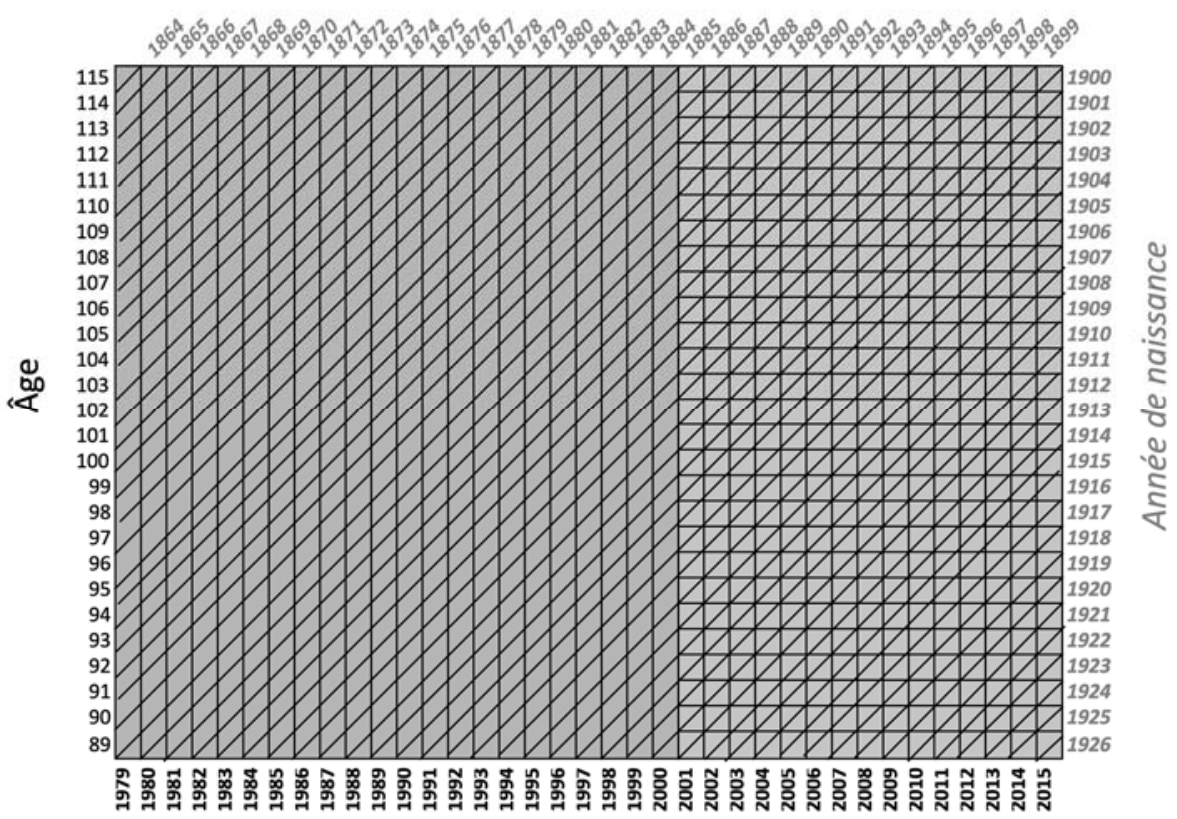

Année de calendrier 
Pour traiter ces données par âge, il nous faut tenir compte de la différence du paramétrage appliqué par l'INSERM selon la période. De 1979 à 1999, il s'agit d'enregistrements individuels de décès par année de naissance et année de décès alors que de 2000 à 2015 ce sont des enregistrements de décès par date exacte de naissance et date exacte de décès. Dans le premier cas, on ne peut donc calculer que des «risques perspectifs» entre deux premiers janviers encadrant une année de décès (Figure 1 : parallélogrammes verticaux du diagramme de Lexis) alors que dans le second cas on peut calculer aussi bien des risques par année d'âge et année de calendrier que des risques perspectifs ou des risques par génération en année d'âge. Nous avons donc construit dans les deux cas des risques perspectifs annuels pour travailler sur des données homogènes.

La seconde différence est que les causes de décès sont classées selon la CIM-9 pour la première période et selon la CIM-10 pour la seconde. Nous utilisons donc de grands groupes de causes les moins perturbés possible par le passage de la 9ème à la 10ème révision de la $\mathrm{CIM}^{2}$. Les huit groupes de causes suivants, à peu près équivalents dans les deux révisions de la CIM (voir en Annexe 1 le tableau des correspondances), sont ainsi considérés ici :

- maladies infectieuses et respiratoires,

- tumeurs,

- démences et maladies neuro-dégénératives (Alzheimer et Parkinson),

- maladies du cœur,

- autres maladies de l'appareil circulatoire,

- toutes autres maladies,

- morts violentes,

- sénilité.

Les maladies et causes de décès mal définies ${ }^{3}$ autres que la sénilité ont été réparties proportionnellement entre les 7 premiers groupes (hors sénilité).

Pour le calcul des taux par âge et par cause, nous avons rapporté les décès aux «populations à risque» utilisées dans la Human Mortality Datadase (HMD) et aimablement communiquées par Magali Barbieri.

2. Le travail de reconstruction de séries temporelles cohérentes suivant la liste détaillée de la Classification internationale des maladies et causes de décès (CIM) entreprise pour la France dans les années 1980 (Vallin, Meslé, 1988) s'arrétait à la CIM-8. Il a été prolongé ensuite pour couvrir la période régie par la CIM-9 (Meslé, Vallin, 1996) mais la prolongation à la CIM-10 est encore inachevée. Elle devrait l'être prochainement dans le cadre de la publication de la base de données HCD (www.causesofdeath.org/cgi-bin/main. php).

3. Chapitres 16 dans la CIM-9 et 18 dans la CIM-10. 


\section{L'impact de la canicule}

La Figure 2 représente pour chaque sexe l'évolution de l'espérance de vie à 90 ans de 1979 à 2017 (Tableau-Annexe 2). Beaucoup plus élevée chez les femmes que chez les hommes, elle a néanmoins, au total, tout autant augmenté chez les premières (de 3,8 ans à 5) que chez les seconds (de 3,2 ans à 4,2). Cependant, dans les deux cas, la canicule marque une forte rupture dans cette évolution et semble déboucher sur de nouveaux rythmes de progrès, accéléré chez les hommes et freiné chez les femmes. Le premier fait, la rupture, est le plus flagrant (Figure 2). On voit non seulement la baisse de l'espérance de vie de 2003, nettement plus forte pour les femmes que pour les hommes, mais on voit aussi très clairement que le niveau recouvré en 2004 se situe, tant pour les femmes que pour les hommes, nettement au-dessus de celui qu'on aurait attendu de la poursuite de la tendance observée entre 1979 et 2002 (premières lignes pointillées). Cependant, le second fait est peut-être plus important pour l'avenir. Alors que chez les hommes l'espérance de vie continue de progresser au-delà de 2004 à un rythme un peu plus soutenu qu'avant 2003, cette progression est fortement freinée chez les femmes au point que la droite de régression après 2004 croise celle d'avant 2003 dès 2015 (droites d'ajustement en pointillé). Autrement dit, ce coup de frein aurait annulé chez les femmes le rebond de vitalité obtenu grâce aux nouvelles attentions portées aux plus âgés alors que ce bonus se serait au contraire amplifié chez les hommes.

Cependant, à regarder de plus près l'évolution qui a précédé la canicule, on peut aussi considérer qu'il y a eu un changement de rythme, surtout chez les femmes, dès le milieu des années 1990. Nous avons donc ajouté deux droites d'ajustement à la Figure 2 (en traits pleins), l'une pour suivre le rythme d'accroissement rapide de la première période pré-caniculaire (1978-1992) et l'autre le rythme ralenti de la seconde (1992-2002). Dans le premier cas, on rejoint chez les hommes des niveaux et une tendance très proches de la réalité observée après la canicule mais pour les femmes on croise au contraire l'ajustement post-caniculaire dès le lendemain de la canicule. Dans le second cas, chez les hommes, l'ajustement sur les années 1992-2002 aboutit en période post-caniculaire à des niveaux beaucoup plus bas et à un rythme nettement moins soutenu que la réalité observée. Et il en va presque de même chez les femmes : bien que le rythme extrapolé soit un peu plus soutenu que le rythme réellement observé, il n'ouvre plus la voie qu'à un croisement lointain, nullement assuré. De toute évidence, dans les deux cas, le changement de tendance intervenu au début des années 1990 préfigure presque parfaitement le rythme post 
caniculaire. Il serait donc injustifié d'attribuer à la canicule elle-même ce changement de rythme. Que s'est-il donc passé ?

Figure 2 Évolution de l'espérance de vie à 90 ans selon le sexe, de 1979 à 2015 (Données chiffrées en Annexe 2)

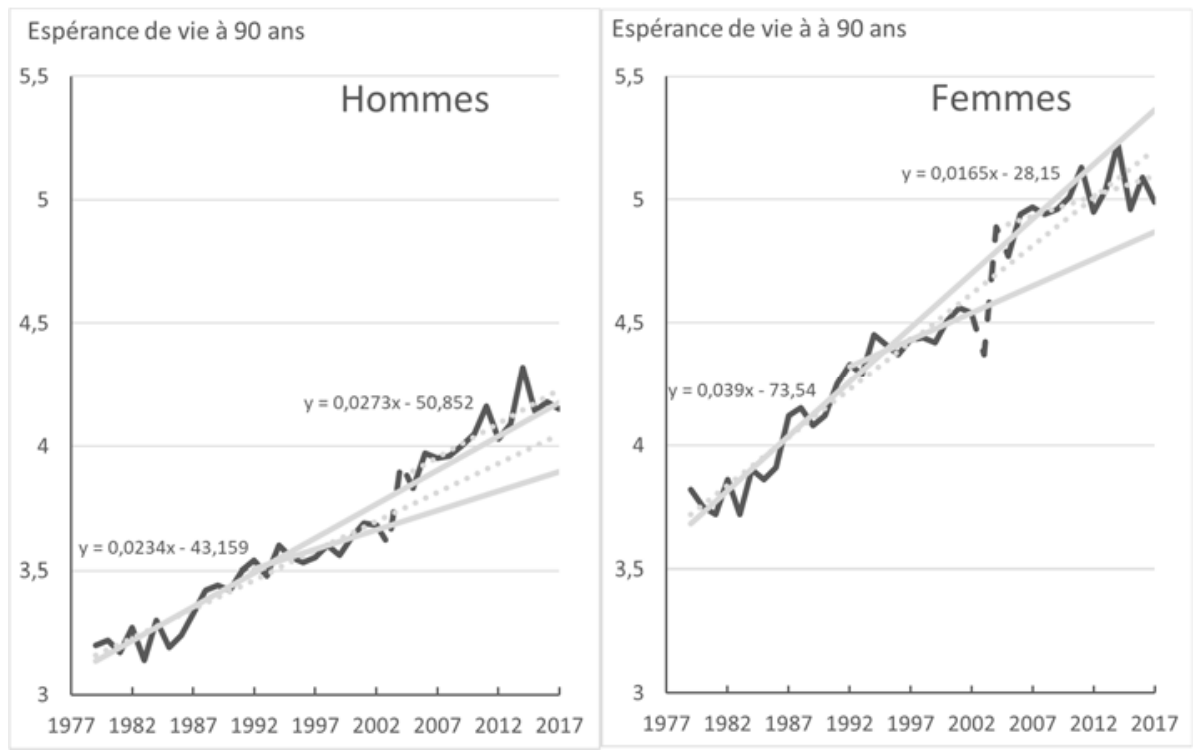
Source : HMD.

Les évolutions par âge (Figure 3) ne nous apprennent pas grand-chose mais plaident dans le même sens. Les baisses les plus importantes ont eu lieu aux âges les plus «jeunes» (90,91, 92 ans, etc.). À mesure qu'on s'élève en âge, la baisse tarde à se manifester notamment chez les hommes, pour lesquels le faible nombre des survivants rend illisible l'évolution des taux trop secoués par les fluctuations aléatoires. Aux âges où elle apparaît clairement, la baisse démarre nettement plus tôt chez les femmes que chez les hommes. Mais elle est aussi plus brutalement interrompue chez ces dernières. En 2004, les deux sexes ont bénéficié d'une retombée du taux plus ample que n'avait été sa montée en 2003 mais, à partir de là, les taux masculins baissent plus vite que les taux féminins.

Pour schématiser l'histoire de ces 39 années d'observation, on doit donc distinguer quatre périodes :

- Une période de vifs progrès (1979-1992) nettement plus marqués chez les femmes avec un gain de 0,51 an (soit 0,5 mois par an) que chez les hommes (0,3 mois par an) ; 
- Une période de ralentissement (1992-2002), là encore, plus nette chez les femmes (tombé de 0,5 mois/an à 0,2) que chez les hommes (de 0,3 mois/an à 0,16$)$;

- La parenthèse caniculaire : 2003-2004, chute de 2003 et rebond de 2004 audelà de la simple récupération, pour les deux sexes ;

- Après la canicule : 2005-2017, l'espérance de vie à 90 ans n'a gagné que 0,22 an chez les femmes $(0,2$ mois par an) contre 0,32 an chez les hommes $(0,4$ mois par an).

Figure 3 Évolution des taux de mortalité par âge à 90 ans et plus selon le sexe, de 1979 à 2015

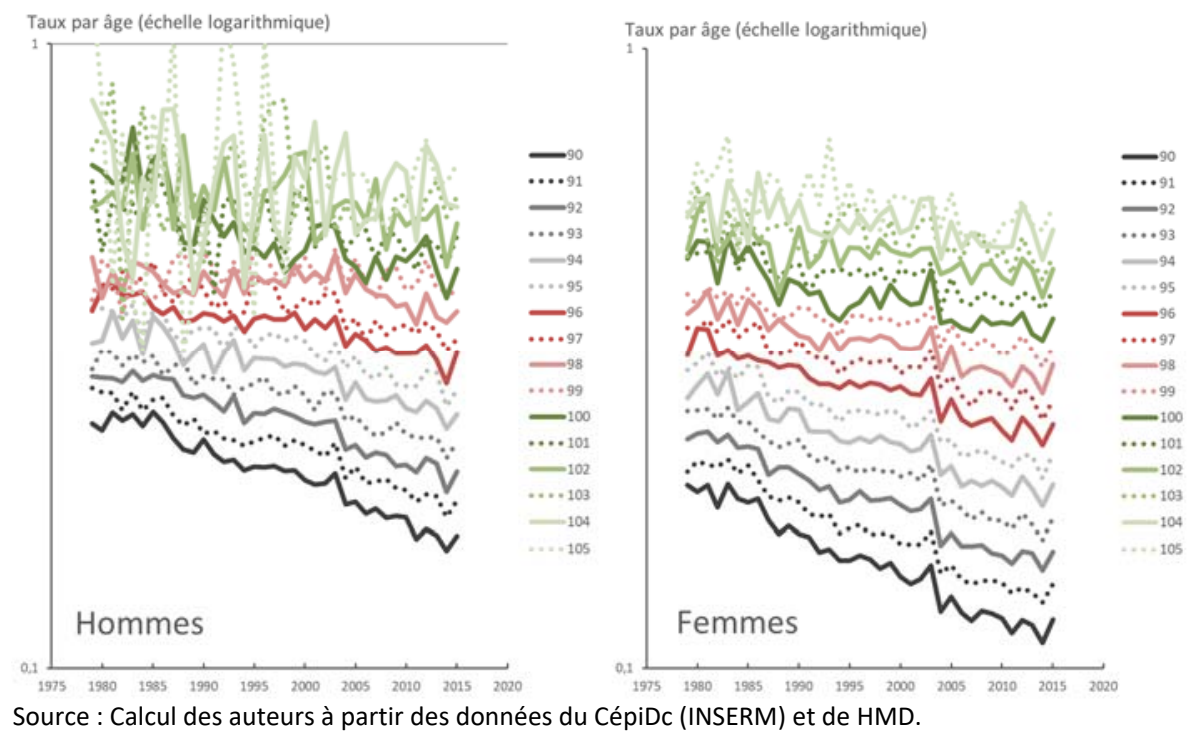

La Figure 4 illustre, en termes d'âge et de causes de décès, l'impact de la crise caniculaire en prenant pour exemple les femmes, les plus fortement touchées tant par la chute que par le rebond (les résultats chiffrés sont donnés en Annexe 3). En 2003, la vague de chaleur a fait perdre aux femmes 0,17 an d'espérance de vie à 90 ans. En 2004, le rebond leur en a fait gagner 0,52 an, trois fois plus ! Sur la Figure 4, les surfaces de couleurs sont, à chaque âge, proportionnelles aux conséquences de l'évolution de chaque groupe de causes et apparaissent en négatif s'il s'agit de pertes et en positif pour les gains, chaque couleur indiquant la cause responsable de la perte ou du gain. 
Figure 4 Poids des 8 grands groupes de causes de décès dans les changements d'espérance de vie à 90 ans avant et après la canicule de 2003, chez les femmes (Voir données chiffrées en Annexe 3)

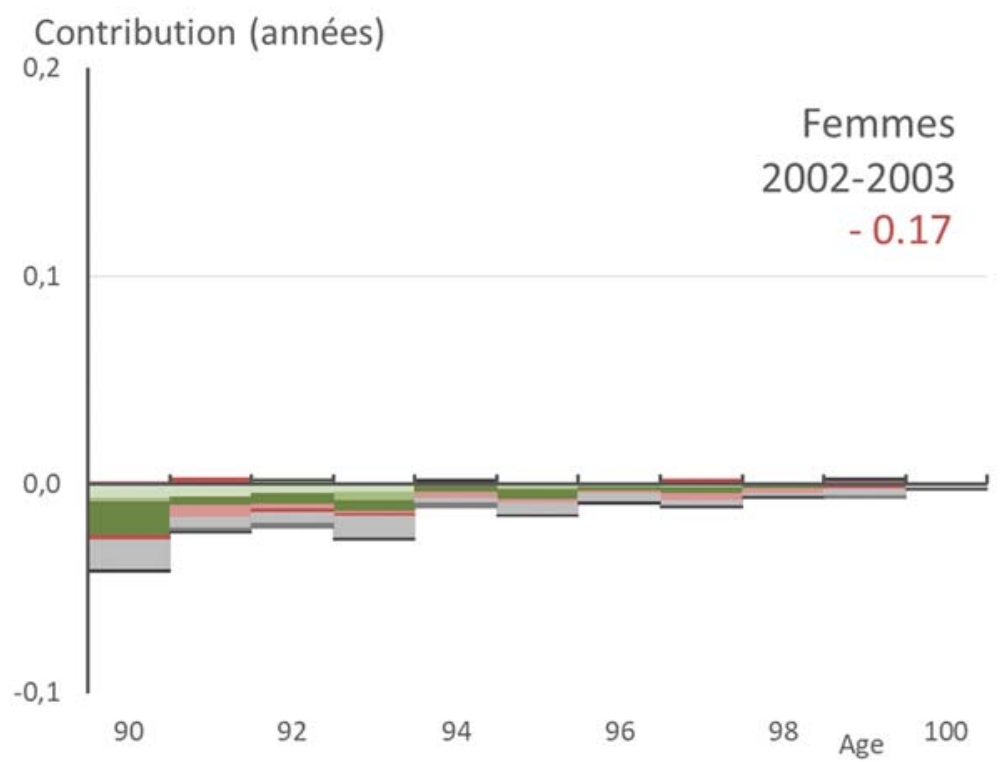

Contribution (années)

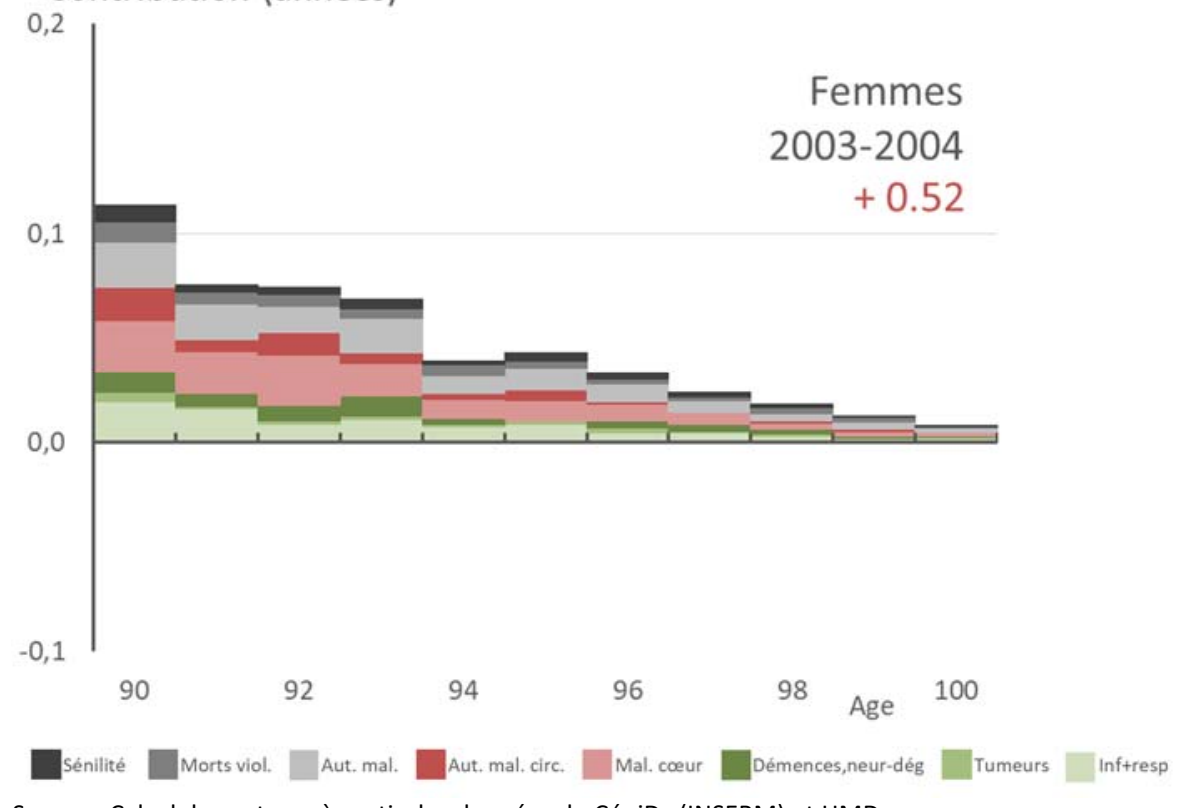

Source : Calcul des auteurs à partir des données du CépiDc (INSERM) et HMD. 
De 2002 à 2003, presque tous les groupes de causes ont pesé négativement à tous les âges. Deux groupes de causes ont joué un rôle particulièrement important sur la baisse de l'espérance de vie : les «démences et maladies neuro-dégénératives» et les «autres maladies». Mais ces conséquences négatives apparaissent finalement comme peu de chose comparées au rebond de 2004. Et ce qui frappe encore plus c'est que les groupes de causes contribuant le plus à ce rebond sont très différents de ceux qui avaient causé les pertes. Les «maladies du cœur» viennent en premier, suivies de près par les «maladies infectieuses et respiratoires» puis par les «autres maladies» et les «autres maladies de l'appareil circulatoire». Les «démences et maladies neuro-dégénératives» n'arrivent plus qu'en cinquième position, à égalité avec les «morts violentes» et la «sénilité». Même le rôle des «tumeurs» n'est plus tout à fait négligeable. On voit ici à quel point, la canicule qui a certes aggravé la mortalité pour certaines causes habituelles chez les vieillards a aussi entraîné une réaction positive des institutions et des acteurs de la santé qui, au-delà de la limite ainsi apportée aux effets de la crise, a plus encore fait reculer dans tous les domaines des processus morbides pour lesquelles il suffisait de plus d'attention au quotidien pour en empêcher ou en retarder l'issue fatale.

\section{Tendances à long terme : continuités et ruptures dans le rôle des causes de décès}

On vient de le voir, la crise caniculaire n'a pas complètement changé le cours des évolutions de mortalité par cause aux âges élevés mais elle a, sinon modifié le rythme de progression, du moins brusquement haussé le niveau de l'espérance de vie à 90 ans et ce, de manière assez différente selon le sexe. Examinons donc ce qui s'est passé avant 2003 et ce qui se passe depuis 2005, séparément pour chaque sexe et en distinguant les deux périodes pré-caniculaires que sépare le changement de rythme du début des années 1990. Nous examinerons ensuite ce qu'il en résulte pour la différence hommes-femmes. 
Gains et pertes d'espérance de vie à 90 ans au cours des trois périodes pré-et post-caniculaires

Du côté des femmes

Reprenant la même représentation graphique qu'à la Figure 4, la Figure 5 (données chiffrées en Annexes 4 et 5) illustre pour chaque grand groupe de causes ce que les femmes ont gagné ou perdu en espérance de vie à 90 ans du fait de l'évolution de la mortalité à chaque âge dans les deux périodes qui ont précédé la canicule (1979-1992 et 1992-2002) et dans celle qui l'a suivie (2005-2015). Cette dernière s'arrête malheureusement ici à 2015 et non 2017, car la statistique par causes est toujours, en France, très en retard sur la statistique toutes causes. Chacune de ces trois périodes est d'une dizaine d'années, ce qui facilite grandement les comparaisons. La Figure 5 nous montre que la dynamique des causes de décès a fortement varié d'une période à l'autre (données chiffrées en Annexe 4).

Figure 5 Poids des 8 grands groupes de causes de décès dans l'évolution de l'espérance de vie à 90 ans des femmes au cours des deux périodes pré-caniculaires et de la période post-caniculaire (Voir données chiffrées en Annexe 4)

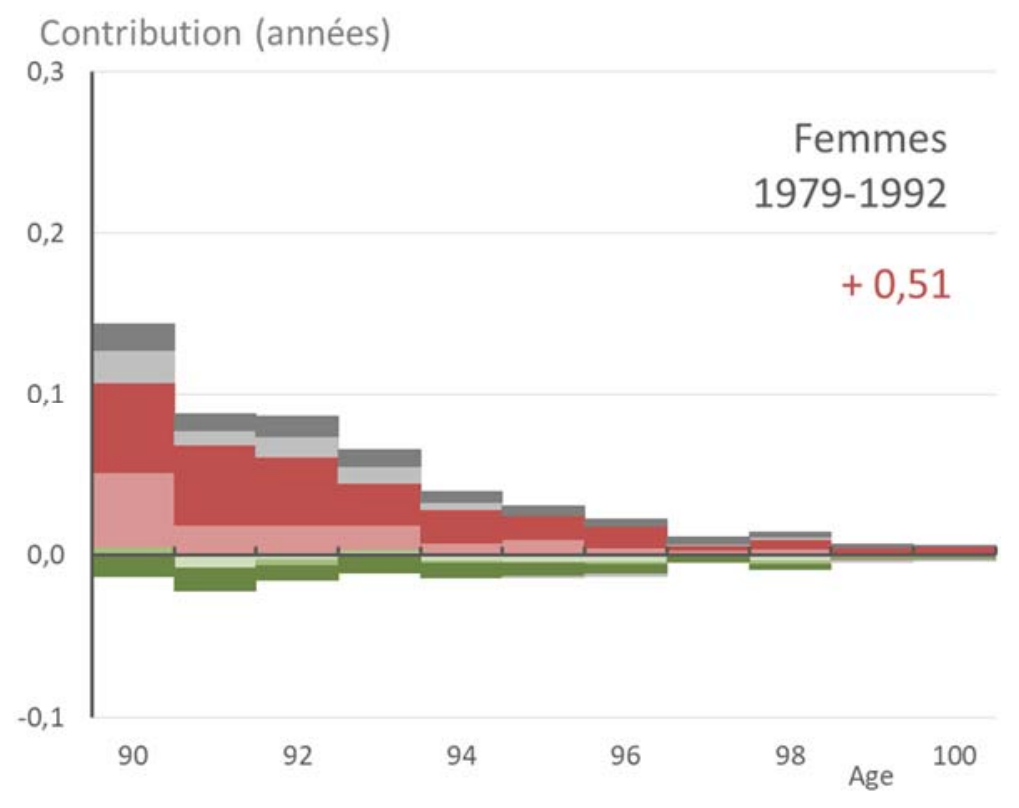



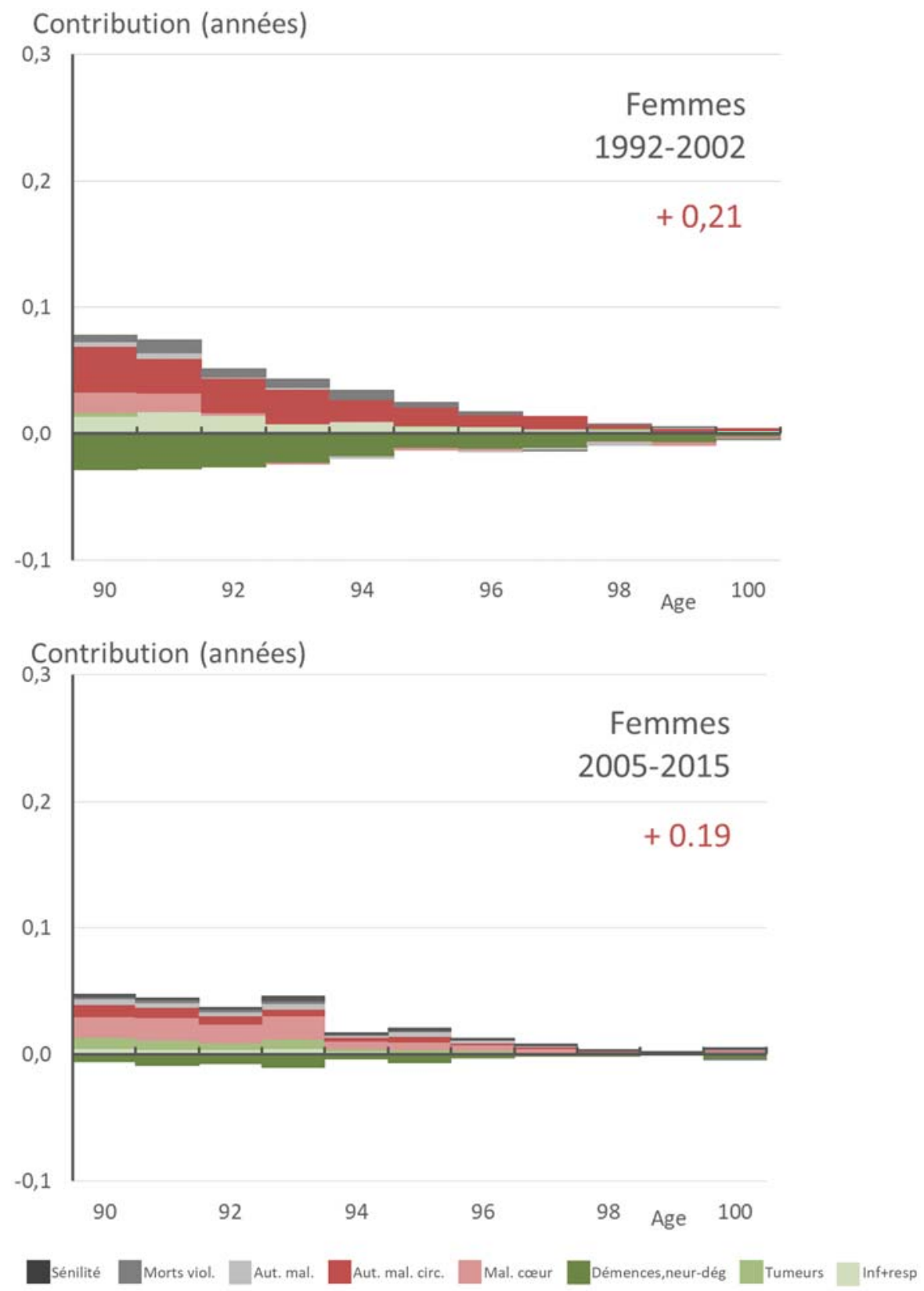

Source : Calcul des auteurs à partir des données du CépiDc (INSERM) et de HMD.

En premier lieu les effets négatifs, presque exclusivement dus aux «démences et maladies neurodégénératives», relativement minimes lors de la première et de la troisième période, sont nettement plus importants 
dans la seconde période. La flambée de ces maladies est même la principale caractéristique nosologique de cette période. Mais il faut aussi souligner que, d'une période à l'autre, non seulement l'importance des effets positifs est variable mais les structures par cause sont assez différentes. La réduction des maladies du cœur, importante à tous les âges en première période, ne touche plus en seconde période que les deux premières classes d'âge (90 et 91 ans) et assez modestement, puis se généralise à nouveau en troisième période. Plus contrastés encore, les effets de la réduction des autres maladies de l'appareil circulatoire sont massifs à tous les âges pendant la première période, alors qu'ils deviennent plus modestes en seconde période et finalement disparaissent en troisième période. De la même façon le recul des morts violentes et des «autres maladies» qui produit encore des effets positifs notables en première période pèse de moins en moins jusqu'à devenir imperceptible en troisième période. En revanche, les effets d'un recul des maladies infectieuses et respiratoires ne sont perceptibles et de façon assez sensible, d'ailleurs, qu'en seconde période, tandis que ceux, beaucoup plus modestes, d'un recul du cancer ne le sont qu'en troisième période.

Enfin, dernière différence majeure, alors qu'en première période l'essentiel des effets, positifs, sur l'espérance de vie était observé à l'âge le plus «jeune» (90 ans) et s'amenuisait ensuite avec l'âge, en seconde période, il n'y a plus de différence entre 90 et 91 ans et en troisième période, plus de différence entre 90, 91, 92 et 93 ans. Autrement dit, le progrès se fait certes plus modeste avec le temps mais concerne des âges de plus en plus élevés.

Au total, en troisième période, ce sont les maladies de cœur qui dominent les progrès de l'espérance de vie, alors qu'en première période, c'était les «autres maladies de l'appareil circulatoire» qui jouaient le plus grand rôle. Entretemps, dans la seconde période le haut de l'affiche a été tenu conjointement par les «autres maladies de l'appareil circulatoire» en positif et par les «démences et maladies neurodégénératives» en négatif.

\section{Du côté des hommes}

Comme pour les femmes, les rôles respectifs des évolutions par cause ont beaucoup changé d'une période à l'autre et l'essentiel de ce qui a été dit pour les femmes se retrouve aussi chez eux. Certains aspects doivent cependant être nuancés. D'une part, les «démences et maladies neurodégénératives» ne sont pas les seules productrices d'effets négatifs sur l'espérance de vie à 90 ans. En première comme en deuxième période, il faut $\mathrm{y}$ ajouter les tumeurs et en première période les maladies infectieuses et 
respiratoires. Quant aux «démences et maladies neurodégénératives» elles-mêmes, l'importance de leurs effets négatifs en deuxième période est moins massive que chez les femmes (données chiffrées en Annexe 5). Le rôle positif des «autres maladies de l'appareil circulatoire», presque imperceptible chez les femmes en troisième période, est beaucoup plus net chez les hommes, de même que celui des cancers à 90-91 ans. D'une manière générale, les contributions positives à l'espérance de vie sont nettement plus marquées chez les hommes que chez les femmes pour cette dernière période. De fait, l'augmentation de l'espérance de vie à 90 ans qui, chez les femmes était passée de 0,51 an en première période à 0,21 an en deuxième période puis à 0,19 an en troisième période est bien tombée, chez les hommes, de 0,34 an en première période à 0,14 an en deuxième période, mais elle est remontée à 0,31 an en troisième période.

Figure 6 Poids des 8 grands groupes de causes de décès dans l'évolution de l'espérance de vie à 90 ans des hommes au cours des deux périodes pré-caniculaire et de la période post-caniculaire (Voir données chiffrées en Annexe 5)

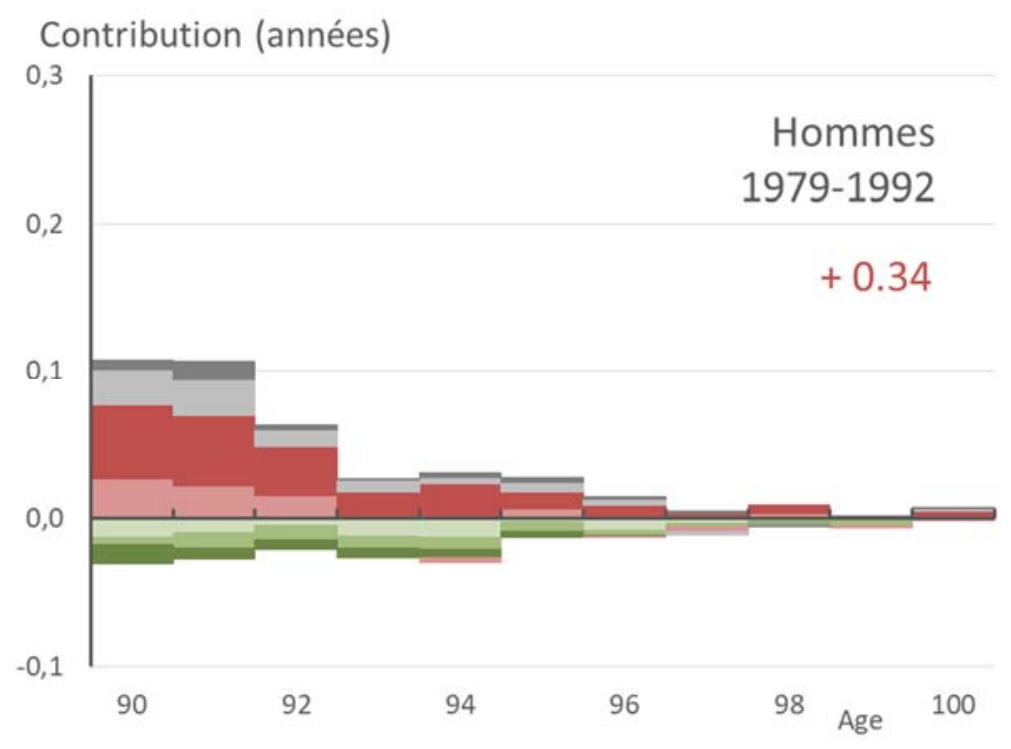




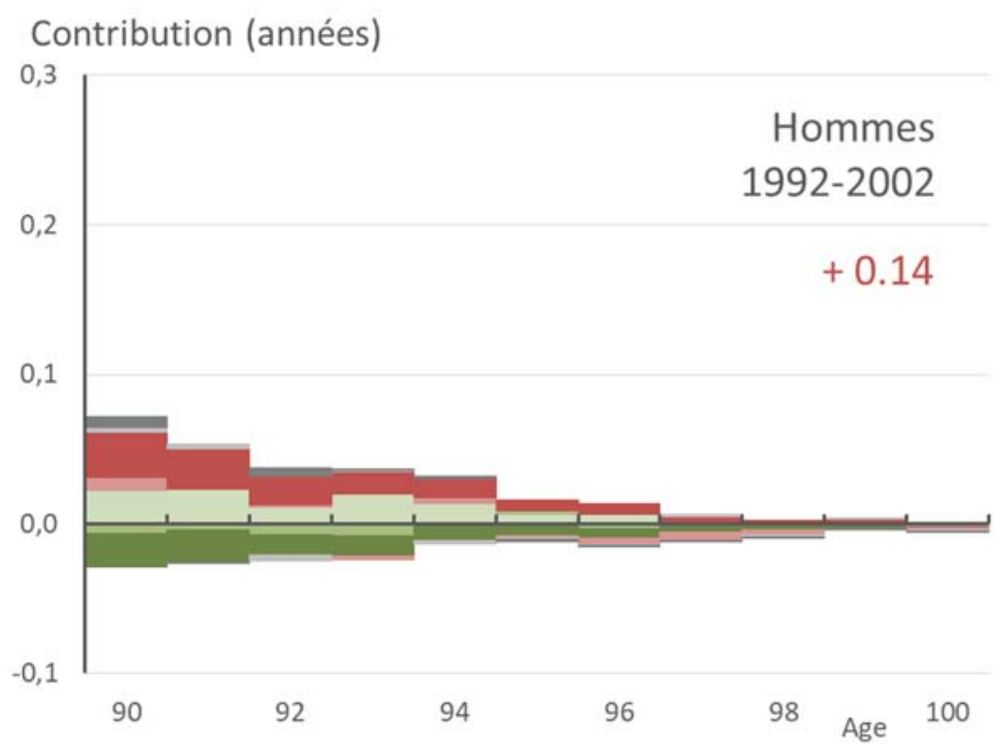

Contribution (années)

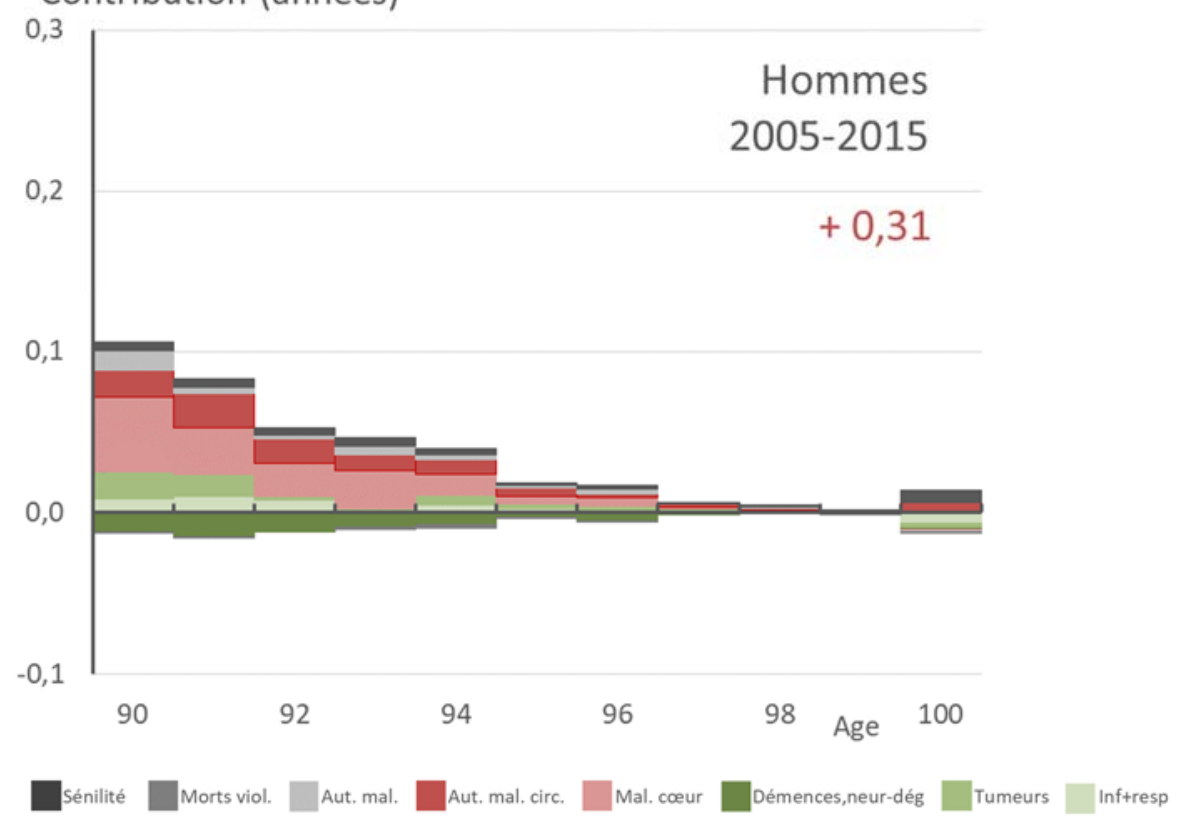

Source : Calcul des auteurs à partir des données du CépiDc (INSERM) et de HMD.

Il y a donc bien eu d'importantes discontinuités tant en niveau qu'en tendance de l'espérance de vie à 90 ans d'une période à l'autre et d'impor- 
tants changements de structure par cause de la mortalité en sont responsables. Cependant ces discontinuités n'excluent pas pour autant toute continuité.

\section{Continuités et discontinuités}

Au contraire, l'examen cause par cause de l'évolution annuelle des taux comparatifs de mortalité montre cependant que les continuités l'emportent sur les discontinuités. À l'échelle de l'ensemble du groupe d'âges 90 ans et plus (Figure 7), la mortalité par «maladies du cœur» domine largement l'ensemble, tant chez les hommes que chez les femmes et elle a fortement diminué chez les deux sexes durant toute la période 1979-2015. Chez les femmes, cette baisse a été très rapide dans la première période pré-caniculaire (1979-1992), puis s'est ralentie dans la seconde (19922002), avant de reprendre de plus belle après la canicule. Chez les hommes elle est restée lente jusqu'en 2003, mais s'est accélérée fortement après la canicule. Certes on ne voit guère chez les hommes d'effet marqué de cette dernière mais cela n'empêche pas que les améliorations du système de santé provoqué par la crise de 2003 puissent être en partie à l'origine de cette accélération.

La continuité est encore plus grande pour les «autres maladies de l'appareil circulatoire», second groupe de causes le plus mortel à la fin des années 1970. La baisse est tout aussi rapide chez les hommes que chez les femmes, et même plus rapide en fin de période, où les progrès se ralentissent pour les femmes. La chute est telle pour ce groupe de causes qu'il tombe de la seconde à la cinquième place en 2015, après les «tumeurs», les «maladies infectieuses et respiratoires», les «autres maladies» et les «démences et maladies neuro-dégénératives». Quoique nettement moins rapides, les baisses de mortalité par «mort violente», «autres maladies» et «sénilité» s'inscrivent aussi dans la continuité.

En revanche, contrairement aux deux principaux changements déjà notés (accélération du recul des «maladies du cœur» chez les hommes, ralentissement du recul des «autres maladies de l'appareil circulatoire» chez les femmes) on note deux ruptures nettes de tendances. La plus importante est certainement le retournement de l'évolution de la mortalité par «tumeurs» chez les hommes, alors que cette mortalité est restée presque parfaitement constante (à un niveau beaucoup plus bas) chez les femmes sur toute la période étudiée ici. Le retournement masculin se produit en 2003-2004. Cependant, lié pour l'essentiel à l'évolution du tabagisme il est sans doute sans grand rapport avec la canicule. En revanche la très forte augmentation du prix du tabac de 2003-2004 a pu conduire nombre 
de fumeurs âgés à cesser de fumer (Dautzenberg, 2012). Le second changement important concerne la mortalité par «maladies infectieuses et respiratoires». Il est surtout marqué chez les hommes qui pâtissaient dans les années 1980 et le début des années 1990 d'une mortalité assez forte, fluctuante et plutôt en augmentation avant de se réduire rapidement à partir du milieu des années 1990. Moins encore que celui de la mortalité par «tumeurs», ce renversement de tendance ne trouve son origine dans la crise caniculaire, puisqu'il lui est antérieur. La crise ne semble même pas avoir accéléré la chute.

FIGURE 7 Évolution des taux comparatifs de mortalité par cause à 90 ans et plus 1979-2015
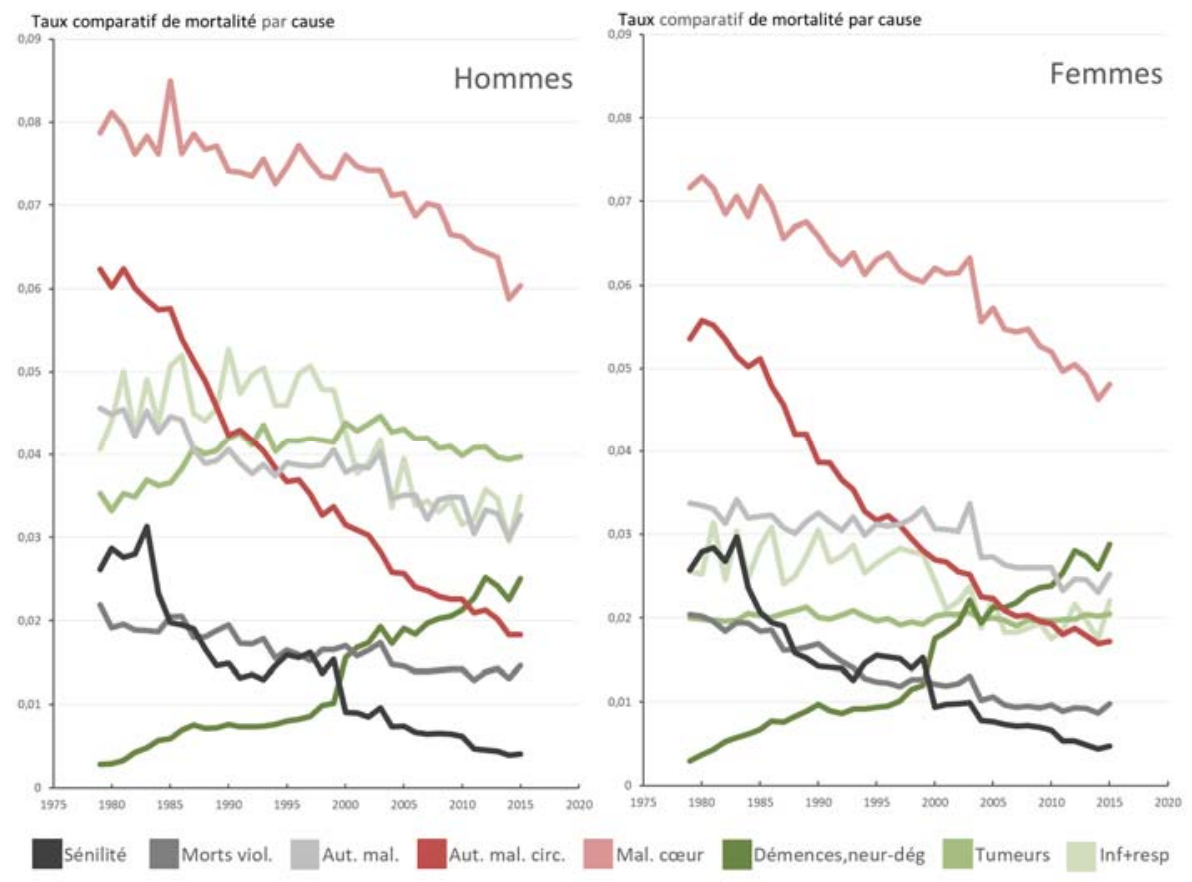

Source : Calcul des auteurs à partir des données du CépiDc (INSERM) et de HMD.

Outre ces observations sur les continuité et discontinuités, il faut aussi noter le croisement assez remarquable des trajectoires de mortalité par «démence et maladies neuro-dégénératives» et par "sénilité». En réalité, une part de l'effet de symétrie observé à la Figure 7 est tout simplement artificielle. En effet, si en 2000 le taux comparatif de mortalité par «démences» augmente brusquement tandis que le taux par «sénilité» dimi- 
nue tout aussi brusquement, c'est tout simplement dû au fait que le passage de la CIM-9 à la CIM-10 a occasionné des échanges trop complexes entre ces deux groupes de causes et avec quelques autres pour être complètement pris en compte dans les regroupements utilisés. Il reste que même si l'on fait abstraction de cet artefact, les trajectoires se croisent car l'usage de la sénilité en tant que cause initiale du décès est devenu moins fréquent tandis que la mortalité par «démence et maladies neurodégénératives» a beaucoup augmenté sous le double effet d'une expansion de ces maladies avec l'augmentation de l'espérance de vie et d'un certain engouement pour la maladie d'Alzheimer. L'impact de la canicule sur ces deux groupes de causes est bien visible.

Les Figures 8a et $8 \mathrm{~b}$ retracent les mêmes trajectoires par groupes de causes pour trois sous-groupes d'âges : 90-92 ans, 93-97 ans et 98 ans et plus. L'idée ici est de comparer les tendances observées chez les 90-92 ans qui, comptent le plus pour le progrès de l'espérance de vie à 90 ans (du moins dans les deux premières périodes), au groupe d'âges le plus élevé rassemblant un nombre de décès encore suffisant pour faire quelque mesure (98 et + ), le groupe intermédiaire ne servant qu'à vérifier qu'il y a une certaine continuité entre les deux extrêmes. Comme on pouvait s'y attendre les trajectoires de taux à 90-92 ans ressemblent très étroitement à celles de la Figure 7 en raison du poids dominant qu'occupent les personnes de cet âge dans la population totale de 90 ans et plus. Et c'est tout aussi vrai pour les femmes que pour les hommes. La seule exception notable est que les taux de mortalité par sénilité y sont nettement plus faibles.

En revanche, à 98 ans et plus, on observe des trajectoires très différentes. Non seulement, en raison des petits nombres, les fluctuations sont extrêmement fortes chez les hommes et souvent assez fortes chez les femmes, mais on observe aussi de nombreuses différences de tendance générale. Le fait le plus marquant est que ni chez les hommes ni chez les femmes la mortalité par «maladies du cœur» ne tend à diminuer, à l'exception, peutêtre, des toutes dernières années pour les femmes.

Au contraire, la mortalité par «sénilité» s'est effondrée chez les femmes et a très profondément diminué chez les hommes. Il était encore fréquent dans les années 1980 et 1990 que tant au niveau de la déclaration que de la codification de la cause initiale, on privilégie cette cause au détriment de processus morbides plus précis difficiles à diagnostiquer. 
FIGURE 8A Évolution 1979-2015 des taux comparatifs de mortalité par cause pour 3 groupes d'âges (Femmes)
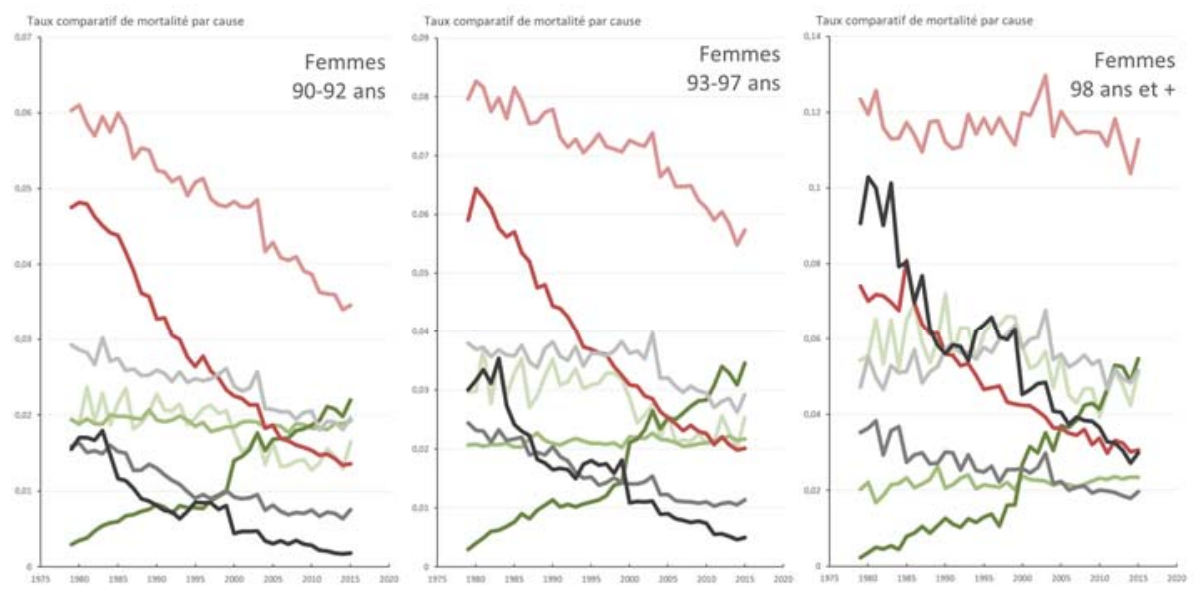

Sénilité

Morts viol. Aut. mal.

Aut. mal, circ. Mal. coeur

Démences,neur-dég Tumeurs Inftresp

Source : Calcul des auteurs à partir des données du CépiDc (INSERM) et de HMD.

Figure 8B Évolution 1979-2015 des taux comparatifs de mortalité par cause pour 3 groupes d'âges (Hommes)
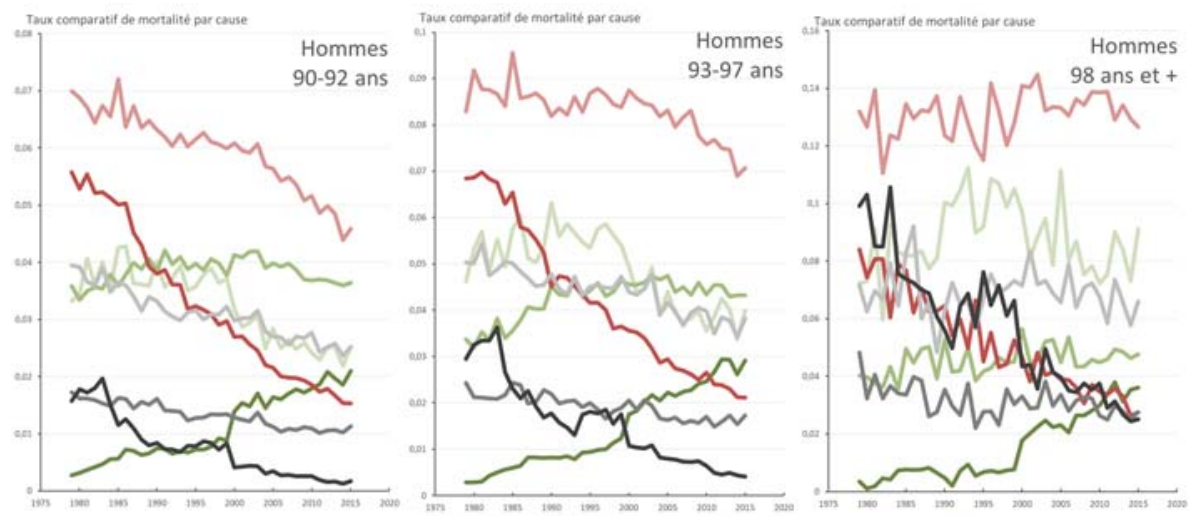

Sénilité

Morts viol.

Aut. mal.

Aut. mal, circ.

Mal. coeur

émences, neur-dég

Tumeurs Inftresp

Source : Calcul des auteurs à partir des données du CépiDc (INSERM) et de HMD.

Entre ces deux visions très différentes des causes de décès aux grands âges, le graphique central des Figures $8 \mathrm{a}$ et $8 \mathrm{~b}$ reflète bien la transition entre un âge (90-92 ans) où les enjeux épidémiologiques apparaissent encore très clairement et le groupe d'âges final (98 ans et plus) où, jusqu'à aujourd'hui, ces derniers restent encore assez flous. 


\section{Renversement de tendance de la différence entre femmes et hommes}

Examinons maintenant les effets de ces tendances par causes assez contrastés sur la différence d'espérance de vie à 90 ans entre femmes et hommes. De 1979 à 2017, l'espérance de vie des hommes à cet âge est passée de 3,2 à 4,2 ans et celle des femmes de 3,8 à 5 ans (Figure 1). Dans le même temps la différence femmes-hommes a évolué de manière assez chaotique, de 0,62 an en 1979 à 0,84 an en 2017, en passant en fait par quatre points bas remarquables dus à deux causes qui ont touché plus sévèrement les femmes que les hommes : la grippe en 1989, en 2015 et en 2017, la canicule en 2003 (Figure 9 et Annexe 6). Si l'on fait abstraction de ces points bas, on constate néanmoins un important changement structurel autour d'un point haut (1,02 an d'écart femmes-hommes) en 2007, précédé d'une montée très nette de la différence et suivi par une baisse dont les grippes de 2015 et 2017 exagèrent peut-être l'ampleur apparente mais ne suffisent pas à l'expliquer entièrement.

FIGURE 9. Évolution 1979-2015 de la différence d'espérance de vie à 90 ans entre femmes et hommes (Données chiffrées en Annexe 2)

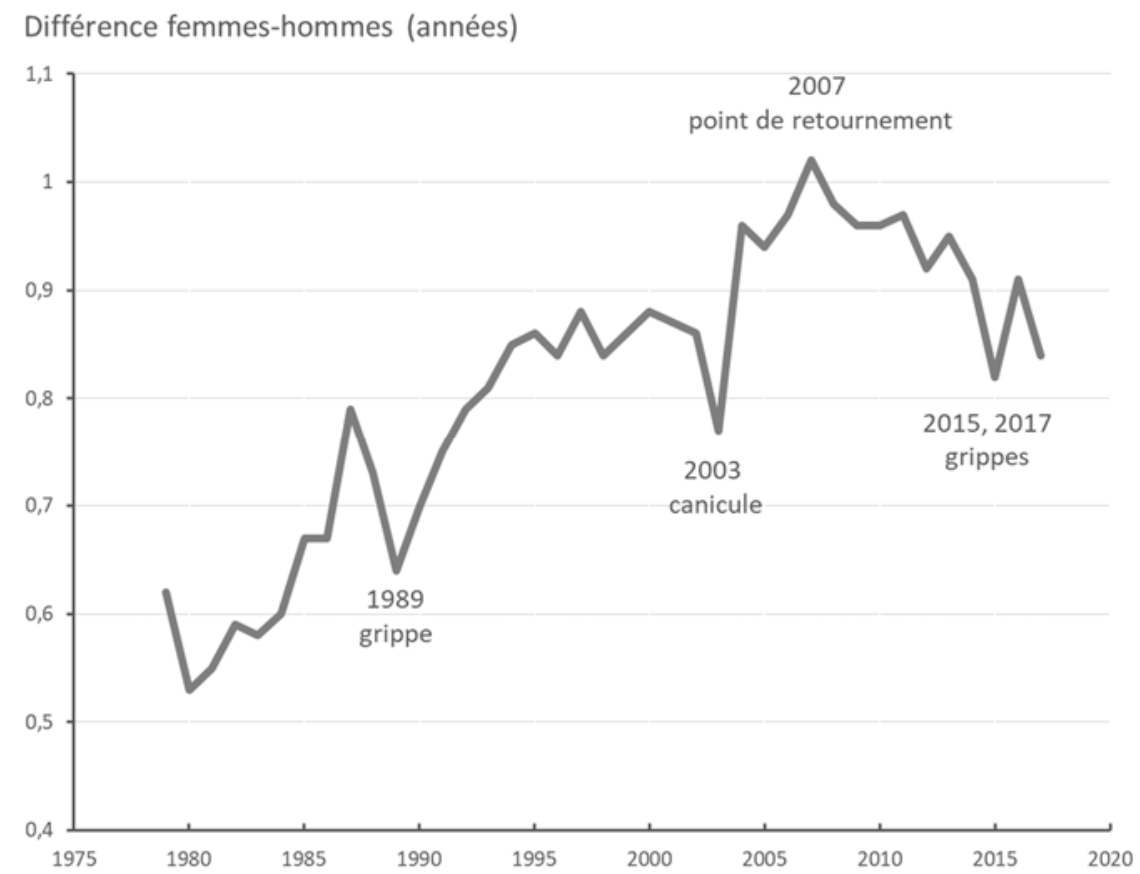

Source : Calcul des auteurs à partir de HMD. 
La Figure 10 illustre la contribution de chaque différence de mortalité par âge et cause de décès à la différence d'espérance de vie en 1979, 2007 et 2015 (voir les données chiffrées en Annexe 6). En 1979, le rôle le plus important était joué par la différence de mortalité par «tumeurs». Venaient ensuite les «maladies infectieuses et respiratoires» et les «autres maladies», puis, assez en retrait, les «maladies du cœur» et les «autres maladies de l'appareil circulatoire». Mais il faut aussi noter le poids relatif énorme des deux premières classes d'âge ( 90 et 91 ans), les classes suivantes $(92,93$ et 94$)$ ne jouant que secondairement sur la différence d'espérance vie, voire presque pas (après 94 ans).

FIGURE 10 Contributions des 8 grands groupes de causes à la différence d'espérance de vie à 90 ans entre femmes et hommes (Données chiffrées en Annexe 6)

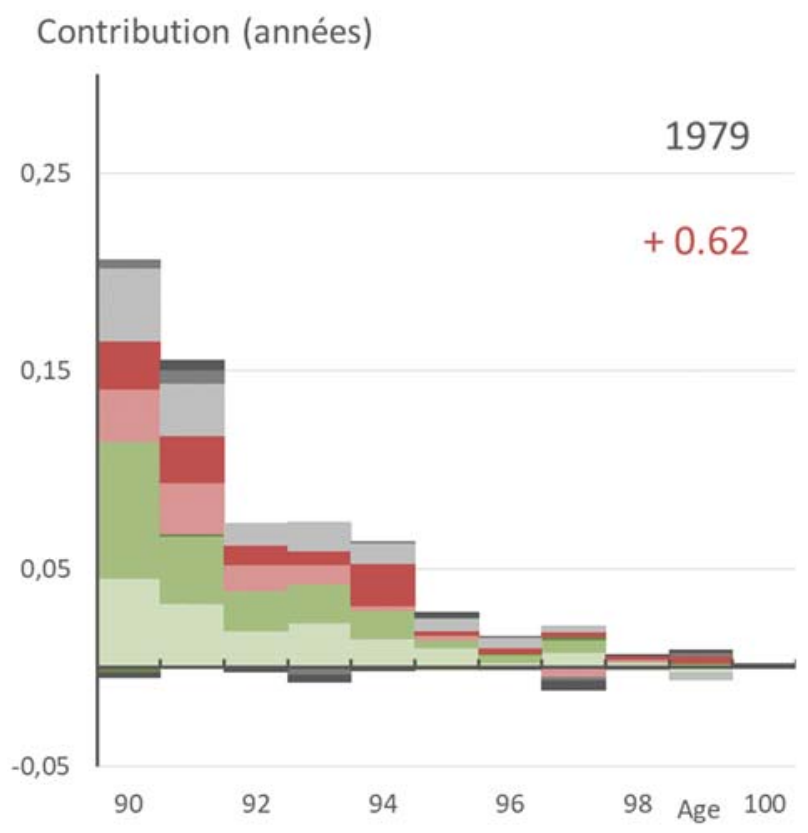




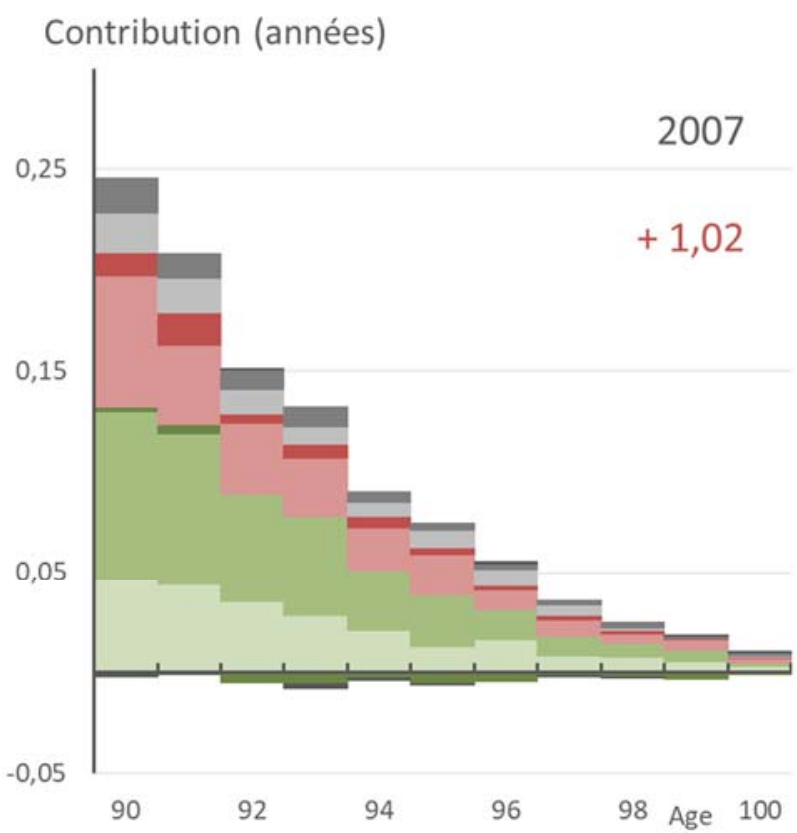

Contribution (années)

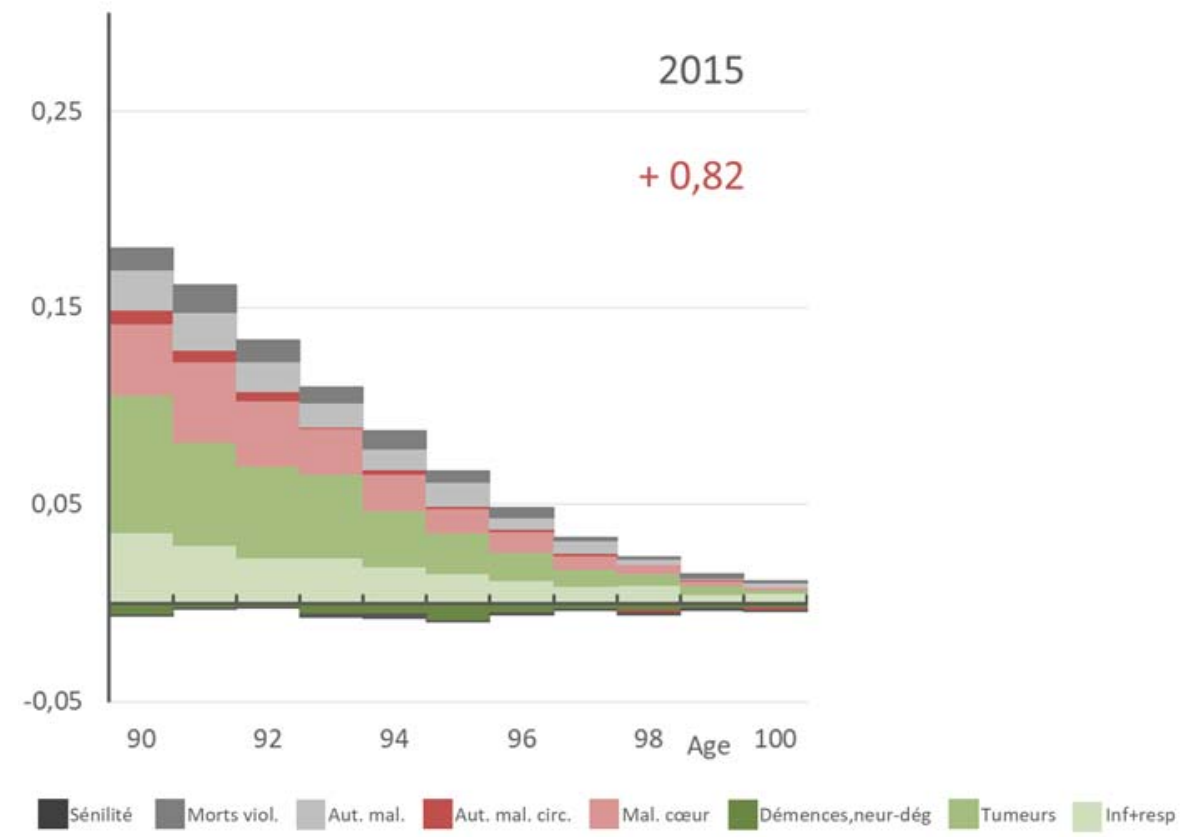

Source : Calcul des auteurs à partir des données du CépiDc (INSERM) et de HMD. 
En 2007, au moment où la différence d'espérance de vie est à son maximum, celle-ci tient toujours essentiellement à la différence de mortalité par «tumeurs» dont le poids s'est accru et surtout s'est beaucoup renforcé à tous les âges supérieurs à 90 ans. Mais l'aggravation de la différence d'espérance de vie tient tout autant à celle de la différence de mortalité par «maladies du cœur» et repose aussi un peu sur celles de la mortalité par «maladies infectieuses et respiratoires» et de la «mortalité violente». En revanche, les rôles des «autres maladies» et des «autres maladies de l'appareil circulatoire» ont sensiblement régressé tandis qu'un des facteurs aggravants a été la montée en puissance au-delà de 91 ans de toutes les différences de mortalité les plus importantes.

En 2015, toutes les causes dont la différence de mortalité dominait la différence d'espérance de vie en 2007 ont sensiblement perdu de leur poids, avec un effet particulièrement visible pour les «maladies infectieuses et respiratoires», du fait de l'épidémie de grippe visiblement défavorable au sexe féminin. Dans le même temps, ces facteurs classiques de la réduction de l'écart d'espérance de vie entre sexes (réduction de la surmortalité masculine) sont renforcés par une surmortalité féminine par «démences et maladies neurodégénératives» qui commence à avoir des effets non négligeables.

\section{Retour sur la qualité des données}

Ainsi l'analyse des causes de décès s'avère-t-elle fort utile pour bien comprendre les tendances et les enjeux épidémiologiques de la mortalité aux grands âges, même au-dessus de 90 ans. Il ne faut pas pour autant penser que la question de la qualité de ces données ne se pose plus. Bien des efforts restent à faire, tant du côté de la certification médicale que de la détermination de la cause initiale du décès.

Nous ne prendrons ici que deux exemples, traités chacun d'un point de vue particulier : les trajectoires comparées de mortalité par «maladies de cœur précisées» et «non précisées» et l'évolution comparée des fréquences par âge de la «sénilité», des «démences» et du groupe «AlzheimerParkinson».

\section{Exemple 1 : Maladies du cœur précisées ou non précisées}

La Figure 11 compare, pour les trois groupes d'âges déjà distingués plus haut (90-92, 93-97 et 98 ans et plus) l'évolution annuelle 1979-2015 des 
taux comparatifs féminins de mortalité par «maladies du cœur précisées» et par «maladies du cœur non précisées» dont la somme correspond au taux comparatif par «maladies du cœur» présenté en Figure 8a. Sur cette Figure 8a seul le total a été utilisé car il nous semblait que les maladies de cœur précisées souffraient encore gravement de sous enregistrement notamment en début de période. Qu'en est-il exactement? On voit clairement ici que les maladies du cœur non précisées (arrêt cardiaque et insuffisance cardiaque), ensemble flou ne regroupant probablement pas seulement des causes directement liées à des pathologies cardiaques, vont en s'amenuisant très rapidement et assez régulièrement sous le double effet de la baisse de la mortalité cardiaque et de l'amélioration progressive des diagnostics. En contrepartie, tant que la part des cas non précisés est importante, le niveau apparent de la mortalité attribuée à des «maladies de cœur précisées» est sous-estimé et stagne ou même augmente à mesure que le taux de l'ensemble non précisé diminue. Les trois graphiques par âge montrent clairement que, plus l'âge est élevé, plus la part des maladies du cœur non précisés est grande, et plus sa réduction a des conséquences importantes sur le taux apparent de mortalité par maladies du cœur précisées ${ }^{4}$. De façon tout à fait logique, à 90-92 ans, les cas précisés dominent dès 1980 et la réduction des cas imprécis provoque seulement une stagnation apparente de la mortalité par «maladies du cœur précisées» et celle-ci baisse à partir du moment où les cas imprécis deviennent très minoritaires. À 93-97 ans, le rôle de l'imprécision joue plus fortement et plus longtemps et en début de période la mortalité par «maladies du cœur précisées» augmente sensiblement jusqu'à ce que la part des imprécisées s'amenuise suffisamment pour que la tendance des maladies précisées s'inverse. Enfin à 98 ans et plus, toujours dans la même logique la mortalité apparente par «maladies du cœur précisées» augmente d'abord très fortement et ne fait que se stabiliser en fin de période.

Bien entendu cette balance entre maladies précisées et non précisées ne commande pas entièrement aux évolutions constatées à la Figure 11. Entre autres choses, l'intervention de la canicule et des réactions à la canicule joue aussi leur rôle, en faisant apparaître dans tous les cas le point haut en 2003. Mais cela ne change rien au principe général. C'est en vertu de ce principe que nous n'avons présenté que le total en Figure 8a et c'est aussi en vertu de ce principe que, si nous voulions traiter de quelques maladies de cœur précises, il nous faudrait traiter les «maladies de cœur

4. C'est ce groupe de causes qui a très certainement bénéficié le plus de l'amélioration des diagnostics, même si cette dernière a eu aussi des effets sur l'évolution de causes non cardiaques. 
non précisées» comme des maladies mal définies et, à tout le moins, tenter de les répartir de la meilleure façon possible entre les maladies précisées pour cerner l'évolution de ces dernières.

Figure 11 Taux comparatifs de mortalité par cause à trois groupes d'âges (Femmes)

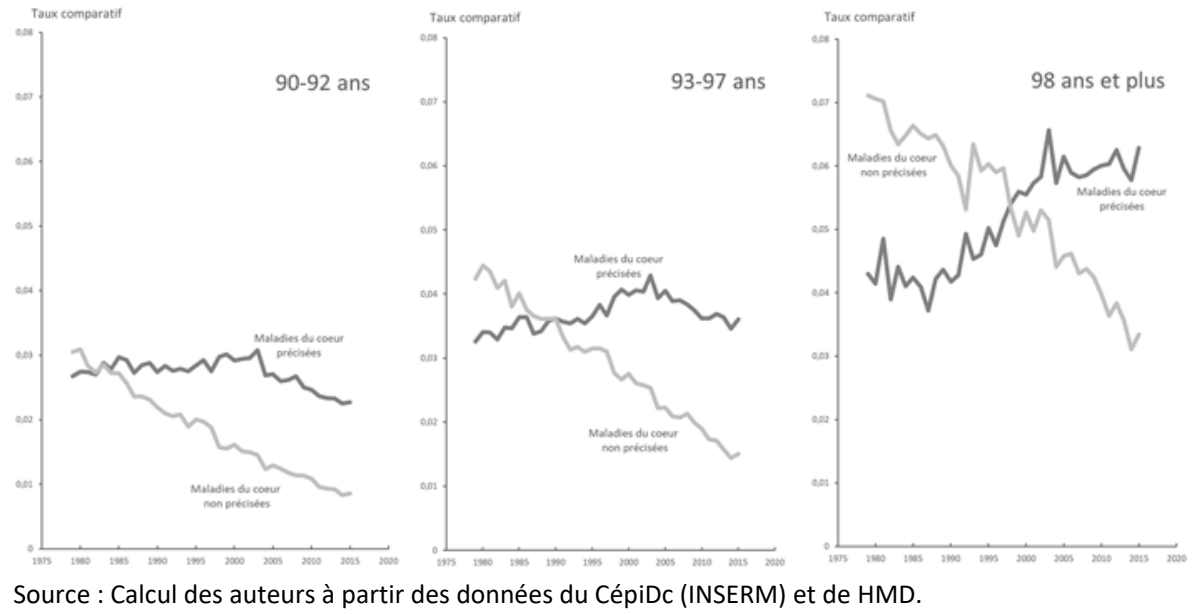

La Figure 11 est aussi source d'espoir quant à la qualité des données, car elle montre assez clairement que, même à ces âges très élevés, le problème des maladies de cœur non précisées est en voie de résorption.

\section{Exemple 2 : Poids relatifs de la sénilité, des démences et du groupe Alzheimer/Parkinson}

Sous un angle tout à fait différent mais également très instructif, la Figure 12 examine la variation avec l'âge des fréquences relatives de trois groupes nosologiques étroitement liés aux très grands âges : la «sénilité», que la CIM range au chapitre des maladies mal définies, les démences (démences vasculaires et démences sans précision), et la somme des deux maladies de dégénérescence neurologique qui ont pris récemment de l'importance (Alzheimer et Parkinson). De 1979 à 2015, la situation a changé du tout au tout (Vallin, Meslé, 1998; Girard, Canestri, 2000). En 1979, cet ensemble nosologique était dominé de façon écrasante par la sénilité. Seules les «démences» trouvaient à ses côtés une certaine existence, encore que bien marginale et limitée au début du groupe d'âges 90 ans et plus, tandis que l'ensemble «maladie d'Alzheimer, maladie de Parkison» 
émergeait à peine et seulement à 90 et 91 ans. En 2015, on observe l'inverse. Les démences occupent une place importante à tous les âges (allant de $40 \%$ à 90 ans à $30 \%$ à 105 ans). «Alzheimer et Parkinson» prennent encore plus de place à 90 ans (50\%) mais, il est vrai, perdent un peu plus de leur importance avec l'âge pour tomber à moins de $20 \%$ après 100 ans. C'est désormais la sénilité qui est réduite à la portion congrue, au moins jusqu'à 100 ans.

Figure 12 Poids relatifs de la sénilité, des démences et du groupe Alzheimer/Parkinson (Femmes)
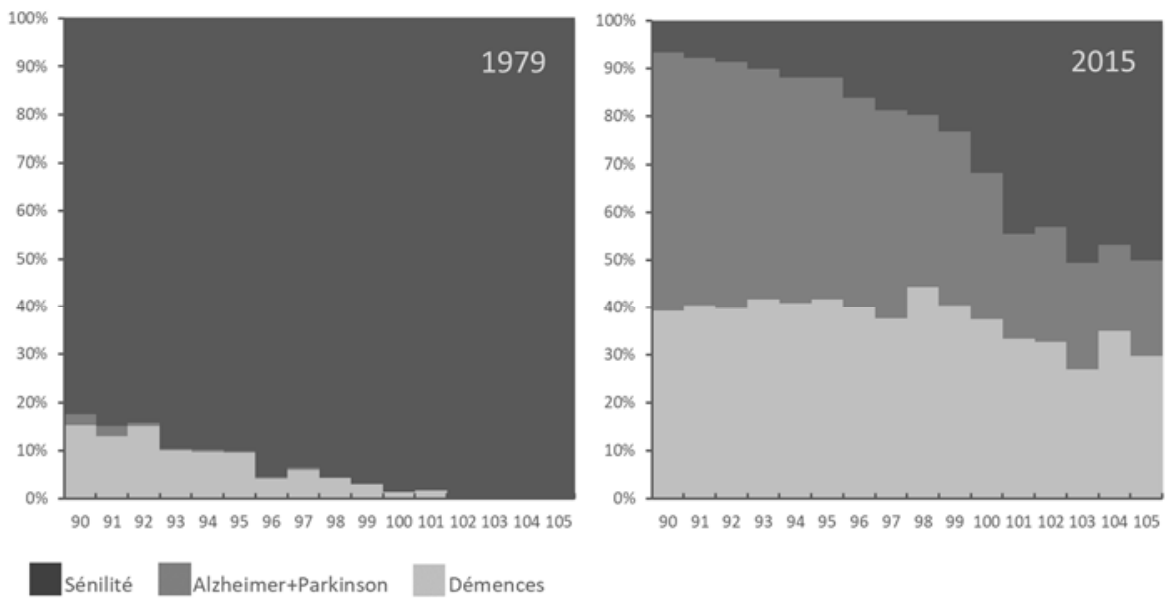

Source : Calcul des auteurs à partir des données du CépiDc (INSERM).

Ce bouleversement de situation est emblématique des précautions que nous avons prises plus haut pour interpréter l'évolution annuelle des groupes «sénilité» et «démences» illustrées par les Figures 7 et 8a.

Il montre aussi que, là encore, il y a bon espoir que les catégories nosologiques utilisées par la statistique acquièrent de plus en plus de sens à l'avenir. On peut même se demander s'il ne conviendrait pas de considérer un peu plus la sénilité comme une vraie cause de décès aux âges très avancés, résumant utilement le fait qu'à ces très grands âges, l'usure générale de l'organisme crée une fragilité indépendante de telle ou telle cause particulière et qu'elle serait, fondamentalement, la vraie cause initiale du décès. C'est en tout cas ce que nous concluions à l'issue d'une étude cas par cas des causes de décès chez les super-centenaires, ces personnes qui ont vécu au moins 110 ans (Meslé, Vallin, 2021). 


\section{Conclusion}

Non seulement l'analyse par cause médicale de la mortalité aux grands âges devient de plus en plus un enjeu de compréhension majeur avec l'allongement de l'espérance de vie, mais cette étude montre aussi qu'elle est devenue possible, même aux très grands âges, et s'avère utile à l'interprétation des changements en cours.

Deux aspects de la mortalité des très vieux ont été mis ici en évidence. Le premier, en apparence anecdotique, les effets de la canicule de 2003, livre un enseignement plus général que ce que l'on peut dire des conséquences directes de cette vague de chaleur. Celle-ci a en effet révélé qu'une plus grande attention au quotidien des personnes très âgées pouvait améliorer sensiblement leur survie en réduisant certaines causes de décès qui ne sont pas forcément celles qu'elle avaient le plus amplifiées («démences et maladies neurodégénératives», «maladies du cœur»), puisqu'en 2004 on a observé un recul important des «maladies infectieuses et respiratoires», des «autres maladies de l'appareil circulatoire», des «autres maladies» des «morts violentes» et de la «sénilité», beaucoup moins affectées en négatif par la canicule.

Plus généralement, on a pu ici rendre compte de la contribution des principaux groupes de causes de décès à la progression de l'espérance de vie à 90 ans au cours des 35 dernières années. Le rôle principal a été joué par le recul spectaculaire des «autres maladies de l'appareil circulatoire» et donc, pour l'essentiel, des maladies cérébro-vasculaires. Le second fait majeur est l'accélération récente de la baisse de la mortalité par «maladies du cœur» mais aussi par «maladies infectieuses et respiratoires». Remarquable aussi est le retournement de la mortalité par «tumeurs» chez les hommes, qui a beaucoup contribué au retournement de la surmortalité masculine elle-même. À l'inverse, la montée du groupe dit «démences» mais qui comprend aussi bien ici la maladie de Parkinson que la maladie d'Alzheimer est réel mais doit être interprété avec prudence en raison des confusions entre ce groupe et la «sénilité» qui, encore forte dans les années 1980, a beaucoup diminué, biaisant un peu les tendances observées.

L'étude a aussi montré que s'il y a bien un avant et un après canicule en termes de niveau général de la mortalité aux âges élevés et notamment de l'espérance de vie à 90 ans, le ralentissement des progrès qui semble caractériser la période post-caniculaire n'est pas lié à la canicule. En fait ce ralentissement était engagé dès la première moitié des années 1990, avec une différence de rythme déjà très nette surtout chez les femmes 
entre les périodes 1979-1992 (croissance très rapide) et 1992-2002 (croissance ralentie). De même, une partie seulement de la différence de structure par cause des progrès réalisés au cours de ces deux périodes et de la période post-caniculaire est liée à la canicule. D'autres sources de changement sont intervenues comme par exemple le renforcement des campagnes anti-tabac au cours de la décennie 1990.

Au-delà du cas de la confusion particulière entre sénilité et démences, cette étude a aussi pointé divers biais causés par le fait qu'en début de période la qualité des données sur les causes de décès laissait encore beaucoup à désirer mais, surtout, qu'elle a depuis fait d'importants progrès. En particulier le diagnostic des «maladies du cœur» aux grands âges a beaucoup gagné en précision tandis que la «sénilité» est de moins en moins utilisée comme un fourre-tout de causes mal définies, au point que l'on peut s'interroger sur la pertinence nouvelle que pourrait acquérir cette notion en tant que reflet de situations où l'usure générale de l'organisme crée une fragilité indépendante de telle ou telle cause particulière, devenant ainsi la vraie cause initiale de la mort.

Ce dernier résultat peut d'ailleurs être mis en parallèle avec le fait que, loin de s'atténuer avec la croissance des effectifs, les fluctuations de la mortalité au très grands âges (98 ans et plus) perdurent quand elles ne tendent pas à augmenter. Fragilisés par cette usure générale, les très vieux ne sont-ils pas de plus en plus à la merci des moindres aléas ?

\section{Références}

DAUTzenberg B. (2012), "Les grandes étapes de la lutte contre le tabagisme en France», Actualité et dossier en santé publique, (81), pp. 38-41.

GiRARD J.-F., CANESTRI A. (2000), La maladie d'Alzheimer, Paris, Direction générale de la santé, Ministère de l'emploi et de la solidarité, Rapport au Gouvernement, 48 p.

GitTelsohn A., Senning J. (1979), "Studies on the Reliability of Vital and Health Records: I. Comparison of Cause of Death and Hospital Record Diagnoses", American Journal of Public Health, 69 (7), pp. 680-689, https://doi.org/10.2105/AJPH.69.7.680.

Hémon D., Jougla E. (2004), Surmortalité liée à la canicule d'août 2003, Paris, INSERM, Rapport remis au Ministre de la Santé et de la Protection Sociale, 75 p.

KanNISto V. (1994), Development of Oldest Old Mortality, Evidence from 28 Developed Countries, 1950-1990, Odense, Odense University Press, Monographs on Population Aging, 1. 
MESLÉ F. (2006), «Causes of Death Among the Oldest-Old: Validity and Comparability", RoBINE J.-M., CRIMmins E. et al. (eds), Human Longevity, Individual Life Duration, and the Growth of the Oldest-Old Population, Dordrecht, Springer, Coll. International Studies in Population, 4, pp. 191-214, https://doi.org/10.1007/978-1-4020-48487_9.

Mesté F., VAluIN J. (1996), «Reconstructing Long-Term Series of Causes of Death: The Case of France", Historical Methods, a Journal of Quantitative and Interdisciplinary History, 29 (2), pp. 72-87, https://doi.org/10.1080/01615440.1996.10112731.

Mesté F., Valuin J. (2021), «Causes of Death at Very Old Age, Including Supercentenarians», MAIeR H., JeUne B., VAUPel J. W. (eds), Exceptional Lifespans, Springer.

Myers G. C., MANTon K. (1983), "Accuracy of Death Certification», Proceedings of the Social Statistics Section, American Statistical Association, pp. 321-330.

OMS (Organisation mondiale de la santé) (1977), Classification internationale des maladies, traumatismes et causes de décès, Neuvième révision, 1, Genève, Organisation mondiale de la santé, $782 \mathrm{p}$.

OMS (Organisation mondiale de la santé) (1993), Classification statistique internationale des maladies et des problèmes de santé connexes, Dixième révision, 1, Genève, Organisation mondiale de la santé, 1335 p.

Rey G., Fouillet A., Jougla É., HÉmon D. (2007), «Vagues de chaleur, fluctuations ordinaires des températures et mortalité en France depuis 1971», Population, 62 (3), pp. 533-563, https://doi.org/10.3917/popu.703.0533.

TOULemon L., BARBIERI M. (2008), «The Mortality Impact of the August 2003 Heat Wave in France: Investigating the 'Harvesting' Effect and Other Long-Term Consequences», Population Studies, 62 (1), pp. 39-53, https://doi.org/10.1080/003247207 01804249.

Valuin J., Meslé F. (1988), Les causes de décès en France de 1925 à 1978, Paris, Ined/PUF, Travaux et Documents, Cahier 115, XX-607 p. + 8 Volumes-Annexes, https://doi.org/10.2307/1533115.

Valuin J., Meslé F. (1998), “Comment suivre l'évolution de la mortalité par cause malgré les discontinuités de la statistique. Le cas de la France de 1925 à 1993», PAVILLON G. (ed), Enjeux des classifications internationales en santé, Paris, Éditions INSERM, Questions en Santé Publique, pp. 113-156. 


\section{Annexes}

TABLEaU 1 Définition des catégories de causes utilisées en fonction des codes de la CIM-9 (OMS, 1977) et de la CIM-10 (OMS, 1993)

\begin{tabular}{l}
\hline Groupe de causes \\
\hline Maladies infectieuses et respiratoires \\
\hline Tumeurs \\
\hline Démences et maladies neuro-dégénératives \\
- démences vasculaires et sans précision \\
- maladies d'Alzheimer et de Parkinson \\
\hline Maladies du cœur \\
- précisées \\
- non précisées \\
\hline Autres maladies de l'appareil circulatoire \\
\hline Toutes autres maladies \\
\hline Morts violentes \\
\hline Sénilité
\end{tabular}

Codes CIM-9

$001-139 ; 460-519$

140-239

290

$3310 ; 332$

$390-4274 ; 4.276-4279 ; 429 \quad 100-145 ; 147-149 ; 151-152$

$4275 ; 428$

$430-459$

$240-299$; 291-330 ; 3311 -

$3319 ; 333-389 ; 520-779$

$800-999$

797
Codes CIM-10

A00-B99 ; J00-J99

C00-D48

F00-F03

G20-G22 ; G30

\author{
$146 ; 150$ \\ G458-G459; 160-199 \\ D50-E90 ; F04-G457 ; \\ G46-H95 ; K00-Q99 \\ V01-Y98
}

R54 
TABleau 2 Espérance de vie à 90 ans

\begin{tabular}{|c|c|c|c|}
\hline \multirow{2}{*}{ Année } & \multicolumn{3}{|c|}{ Espérance de vie } \\
\hline & Hommes & Femmes & Différence \\
\hline 1979 & 3,20 & 3,82 & 0,62 \\
\hline 1980 & 3,22 & 3,75 & 0,53 \\
\hline 1981 & 3,17 & 3,72 & 0,55 \\
\hline 1982 & 3,27 & 3,86 & 0,59 \\
\hline 1983 & 3,14 & 3,72 & 0,58 \\
\hline 1984 & 3,30 & 3,90 & 0,60 \\
\hline 1985 & 3,19 & 3,86 & 0,67 \\
\hline 1986 & 3,24 & 3,91 & 0,67 \\
\hline 1987 & 3,33 & 4,12 & 0,79 \\
\hline 1988 & 3,42 & 4,15 & 0,73 \\
\hline 1989 & 3,44 & 4,08 & 0,64 \\
\hline 1990 & 3,42 & 4,12 & 0,70 \\
\hline 1991 & 3,50 & 4,25 & 0,75 \\
\hline 1992 & 3,54 & 4,33 & 0,79 \\
\hline 1993 & 3,48 & 4,29 & 0,81 \\
\hline 1994 & 3,60 & 4,45 & 0,85 \\
\hline 1995 & 3,55 & 4,41 & 0,86 \\
\hline 1996 & 3,53 & 4,37 & 0,84 \\
\hline 1997 & 3,55 & 4,43 & 0,88 \\
\hline 1998 & 3,60 & 4,44 & 0,84 \\
\hline 1999 & 3,56 & 4,42 & 0,86 \\
\hline 2000 & 3,63 & 4,51 & 0,88 \\
\hline 2001 & 3,69 & 4,56 & 0,87 \\
\hline 2002 & 3,68 & 4,54 & 0,86 \\
\hline 2003 & 3,60 & 4,37 & 0,77 \\
\hline 2004 & 3,93 & 4,89 & 0,96 \\
\hline 2005 & 3,83 & 4,77 & 0,94 \\
\hline 2006 & 3,97 & 4,94 & 0,97 \\
\hline 2007 & 3,95 & 4,97 & 1,02 \\
\hline 2008 & 3,96 & 4,94 & 0,98 \\
\hline 2009 & 4,00 & 4,96 & 0,96 \\
\hline 2010 & 4,05 & 5,01 & 0,96 \\
\hline 2011 & 4,16 & 5,13 & 0,97 \\
\hline 2012 & 4,03 & 4,95 & 0,92 \\
\hline 2013 & 4,09 & 5,04 & 0,95 \\
\hline 2014 & 4,32 & 5,23 & 0,91 \\
\hline 2015 & 4,14 & 4,96 & 0,82 \\
\hline 2016 & 4,18 & 5,09 & 0,91 \\
\hline 2017 & 4,15 & 4,99 & 0,84 \\
\hline & & & \\
\hline
\end{tabular}


TABleau 3 Composantes par âge et cause de la perte d'espérance de vie à 90 ans due à la canicule et de la récupération observée l'année suivante, sexe féminin (Années et millièmes d'années)

\begin{tabular}{|c|c|c|c|c|c|c|c|c|c|}
\hline \multirow[b]{2}{*}{ 总 } & \multicolumn{9}{|c|}{ Grands groupes de causes } \\
\hline & 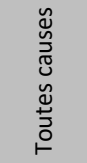 & 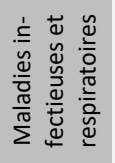 & 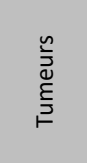 & 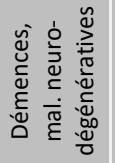 & 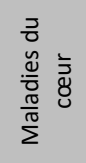 & 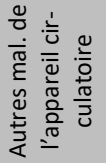 & 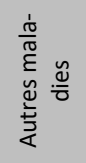 & 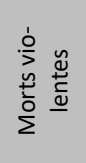 & \\
\hline \multicolumn{10}{|c|}{ Canicule (différence 2002-2003) } \\
\hline 90 & $-0,041$ & $-0,007$ & $-0,002$ & $-0,016$ & 0,001 & $-0,003$ & $-0,014$ & 0,000 & $-0,001$ \\
\hline 91 & $-0,020$ & $-0,006$ & 0,000 & $-0,004$ & $-0,006$ & 0,003 & $-0,005$ & $-0,002$ & 0,000 \\
\hline 92 & $-0,019$ & $-0,005$ & 0,002 & $-0,005$ & $-0,002$ & $-0,002$ & $-0,005$ & $-0,002$ & 0,000 \\
\hline 93 & $-0,027$ & $-0,004$ & $-0,004$ & $-0,005$ & $-0,002$ & $-0,001$ & $-0,011$ & 0,000 & $-0,001$ \\
\hline 94 & $-0,010$ & $-0,001$ & 0,000 & $-0,003$ & $-0,003$ & 0,001 & $-0,002$ & $-0,002$ & 0,001 \\
\hline 95 & $-0,015$ & $-0,002$ & $-0,001$ & $-0,004$ & $-0,002$ & 0,000 & $-0,006$ & 0,000 & $-0,001$ \\
\hline 96 & $-0,010$ & $-0,001$ & $-0,001$ & $-0,002$ & $-0,001$ & 0,000 & $-0,004$ & 0,000 & $-0,001$ \\
\hline 97 & $-0,009$ & $-0,002$ & 0,001 & $-0,003$ & $-0,003$ & 0,001 & $-0,002$ & 0,000 & $-0,001$ \\
\hline 98 & $-0,007$ & 0,000 & $-0,001$ & $-0,001$ & $-0,002$ & 0,000 & $-0,001$ & $-0,001$ & 0,000 \\
\hline 99 & $-0,005$ & $-0,001$ & 0,001 & 0,000 & 0,001 & $-0,001$ & $-0,004$ & $-0,001$ & 0,001 \\
\hline 100 & $-0,002$ & $-0,001$ & 0,000 & 0,000 & 0,000 & 0,000 & $-0,001$ & 0,000 & 0,000 \\
\hline 101 et + & $-0,004$ & $-0,001$ & 0,001 & $-0,018$ & 0,010 & 0,011 & $-0,002$ & $-0,012$ & 0,006 \\
\hline Somme & $-0,170$ & $-0,030$ & $-0,004$ & $-0,062$ & $-0,010$ & 0,011 & $-0,057$ & $-0,021$ & 0,003 \\
\hline \multicolumn{10}{|c|}{ Récupération (différence 2003-2004) } \\
\hline 90 & 0,113 & 0,019 & 0,005 & 0,010 & 0,024 & 0,016 & 0,021 & 0,010 & 0,008 \\
\hline 91 & 0,076 & 0,016 & 0,001 & 0,006 & 0,020 & 0,006 & 0,017 & 0,006 & 0,003 \\
\hline 92 & 0,074 & 0,008 & 0,002 & 0,007 & 0,025 & 0,010 & 0,013 & 0,006 & 0,003 \\
\hline 93 & 0,068 & 0,010 & 0,002 & 0,009 & 0,016 & 0,005 & 0,016 & 0,005 & 0,004 \\
\hline 94 & 0,039 & 0,007 & 0,001 & 0,003 & 0,009 & 0,003 & 0,009 & 0,006 & 0,002 \\
\hline 95 & 0,043 & 0,008 & 0,002 & 0,000 & 0,010 & 0,005 & 0,011 & 0,003 & 0,004 \\
\hline 96 & 0,033 & 0,005 & 0,002 & 0,003 & 0,008 & 0,001 & 0,009 & 0,003 & 0,003 \\
\hline 97 & 0,023 & 0,005 & 0,001 & 0,003 & 0,005 & $-0,001$ & 0,006 & 0,002 & 0,002 \\
\hline 98 & 0,018 & 0,003 & 0,001 & 0,003 & 0,003 & 0,001 & 0,003 & 0,003 & 0,002 \\
\hline 99 & 0,012 & 0,002 & 0,000 & 0,001 & 0,002 & 0,001 & 0,003 & 0,002 & 0,000 \\
\hline 100 & 0,008 & 0,001 & 0,001 & 0,001 & 0,002 & 0,000 & 0,002 & 0,001 & 0,001 \\
\hline 101 et + & 0,012 & 0,003 & 0,000 & 0,000 & 0,004 & 0,000 & 0,002 & 0,002 & 0,002 \\
\hline Somme & 0,520 & 0,087 & 0,016 & 0,048 & 0,128 & 0,049 & 0,112 & 0,047 & 0,035 \\
\hline
\end{tabular}


TABleau 4 Composantes par âge et cause des changements d'espérance de vie à 90 ans observés durant les périodes ayant précédé ou suivi la canicule, sexe féminin (Années et millièmes d'années)

\begin{tabular}{|c|c|c|c|c|c|c|c|c|c|}
\hline \multirow[b]{2}{*}{ 总 } & \multicolumn{9}{|c|}{ Grands groupes de causes } \\
\hline & 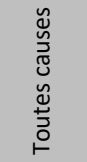 & 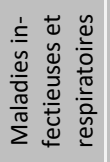 & 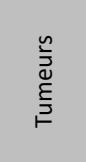 & 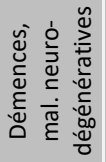 & 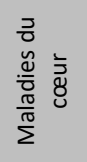 & 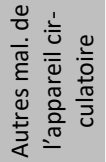 & 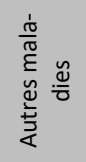 & 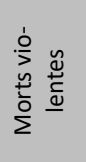 & 离 \\
\hline \multicolumn{10}{|c|}{ Première période avant la canicule (1979-1992) } \\
\hline 90 & 0,146 & 0,001 & 0,004 & $-0,012$ & 0,043 & 0,052 & 0,019 & 0,015 & 0,024 \\
\hline 91 & 0,085 & $-0,008$ & 0,001 & $-0,013$ & 0,017 & 0,046 & 0,009 & 0,009 & 0,024 \\
\hline 92 & 0,084 & $-0,002$ & $-0,003$ & $-0,009$ & 0,018 & 0,039 & 0,012 & 0,011 & 0,018 \\
\hline 93 & 0,068 & 0,002 & 0,002 & $-0,010$ & 0,013 & 0,024 & 0,009 & 0,010 & 0,017 \\
\hline 94 & 0,036 & $-0,003$ & $-0,001$ & $-0,008$ & 0,007 & 0,019 & 0,005 & 0,006 & 0,012 \\
\hline 95 & 0,029 & $-0,004$ & $-0,001$ & $-0,007$ & 0,009 & 0,014 & $-0,001$ & 0,006 & 0,013 \\
\hline 96 & 0,019 & $-0,004$ & $-0,002$ & $-0,005$ & 0,005 & 0,013 & $-0,001$ & 0,004 & 0,010 \\
\hline 97 & 0,015 & 0,001 & $-0,001$ & $-0,003$ & 0,001 & 0,003 & 0,000 & 0,004 & 0,008 \\
\hline 98 & 0,012 & $-0,003$ & $-0,002$ & $-0,003$ & 0,004 & 0,006 & 0,001 & 0,003 & 0,007 \\
\hline 99 & 0,006 & $-0,001$ & $-0,001$ & $-0,001$ & $-0,001$ & 0,004 & $-0,001$ & 0,002 & 0,003 \\
\hline 100 & 0,006 & $-0,001$ & 0,000 & $-0,001$ & 0,002 & 0,003 & $-0,001$ & 0,001 & 0,003 \\
\hline 101 et + & 0,004 & $-0,002$ & 0,000 & $-0,001$ & 0,000 & 0,002 & $-0,003$ & 0,001 & 0,007 \\
\hline Somme & 0,510 & $-0,023$ & $-0,005$ & $-0,073$ & 0,118 & 0,224 & 0,048 & 0,073 & 0,147 \\
\hline \multicolumn{10}{|c|}{ Seconde période avant la canicule (1992-2002) } \\
\hline 90 & 0,053 & 0,013 & 0,002 & $-0,026$ & 0,015 & 0,034 & 0,003 & 0,005 & 0,007 \\
\hline 91 & 0,052 & 0,016 & 0,000 & $-0,026$ & 0,014 & 0,025 & 0,004 & 0,010 & 0,009 \\
\hline 92 & 0,031 & 0,013 & 0,000 & $-0,024$ & 0,002 & 0,025 & 0,001 & 0,006 & 0,008 \\
\hline 93 & 0,027 & 0,008 & $-0,001$ & $-0,021$ & $-0,001$ & 0,025 & 0,002 & 0,006 & 0,009 \\
\hline 94 & 0,020 & 0,009 & $-0,001$ & $-0,017$ & 0,001 & 0,016 & $-0,001$ & 0,007 & 0,006 \\
\hline 95 & 0,017 & 0,006 & 0,000 & $-0,011$ & $-0,001$ & 0,014 & 0,000 & 0,003 & 0,006 \\
\hline 96 & 0,008 & 0,006 & $-0,001$ & $-0,011$ & $-0,001$ & 0,009 & 0,000 & 0,001 & 0,005 \\
\hline 97 & 0,005 & 0,003 & $-0,002$ & $-0,009$ & 0,001 & 0,009 & $-0,001$ & 0,000 & 0,004 \\
\hline 98 & $-0,001$ & 0,003 & 0,002 & $-0,006$ & $-0,001$ & 0,002 & $-0,002$ & 0,000 & 0,001 \\
\hline 99 & 0,000 & 0,001 & 0,000 & $-0,006$ & $-0,002$ & 0,003 & 0,001 & 0,001 & 0,004 \\
\hline 100 & 0,002 & 0,002 & 0,000 & $-0,003$ & $-0,001$ & 0,002 & 0,000 & 0,000 & 0,003 \\
\hline 101 et + & $-0,003$ & 0,002 & $-0,001$ & $-0,003$ & $-0,005$ & 0,003 & $-0,001$ & 0,000 & 0,002 \\
\hline Somme & 0,210 & 0,082 & 0,000 & $-0,164$ & 0,021 & 0,166 & 0,003 & 0,039 & 0,062 \\
\hline \multicolumn{10}{|c|}{ Période après la canicule (2005-2015) } \\
\hline 90 & 0,041 & 0,005 & 0,009 & $-0,006$ & 0,016 & 0,009 & 0,005 & 0,002 & 0,002 \\
\hline 91 & 0,036 & 0,004 & 0,007 & $-0,009$ & 0,018 & 0,008 & 0,004 & 0,002 & 0,002 \\
\hline 92 & 0,030 & 0,004 & 0,005 & $-0,007$ & 0,015 & 0,007 & 0,003 & 0,002 & 0,002 \\
\hline 93 & 0,036 & 0,004 & 0,008 & $-0,010$ & 0,018 & 0,006 & 0,004 & 0,002 & 0,004 \\
\hline 94 & 0,013 & 0,002 & 0,003 & $-0,004$ & 0,006 & 0,003 & 0,002 & 0,001 & 0,002 \\
\hline 95 & 0,014 & 0,002 & 0,002 & $-0,007$ & 0,006 & 0,004 & 0,004 & 0,001 & 0,002 \\
\hline 96 & 0,010 & 0,001 & 0,002 & $-0,003$ & 0,004 & 0,002 & 0,002 & 0,000 & 0,001 \\
\hline 97 & 0,006 & 0,001 & 0,000 & $-0,002$ & 0,003 & 0,001 & 0,001 & 0,000 & 0,001 \\
\hline 98 & 0,002 & 0,001 & 0,000 & $-0,001$ & 0,001 & 0,000 & 0,000 & 0,000 & 0,000 \\
\hline 99 & 0,001 & 0,000 & 0,000 & 0,000 & 0,001 & 0,000 & 0,000 & 0,000 & 0,000 \\
\hline 100 & 0,001 & 0,001 & $-0,001$ & $-0,003$ & 0,001 & 0,001 & 0,001 & 0,000 & 0,002 \\
\hline 101 et + & $-0,001$ & $-0,001$ & 0,000 & 0,001 & 0,000 & 0,000 & 0,000 & 0,000 & $-0,001$ \\
\hline Somme & 0,190 & 0,024 & 0,035 & $-0,051$ & 0,089 & 0,041 & 0,025 & 0,011 & 0,017 \\
\hline
\end{tabular}


TABLEAU 5 Composantes par âge et cause des changements d'espérance de vie à 90 ans observés durant les périodes ayant précédé ou suivi la canicule, sexe masculin (Années et millièmes d'années)

\begin{tabular}{|c|c|c|c|c|c|c|c|c|c|}
\hline \multirow[b]{2}{*}{ 总 } & \multicolumn{9}{|c|}{ Grands groupes de causes } \\
\hline & 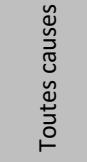 & 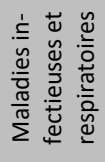 & 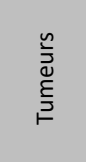 & 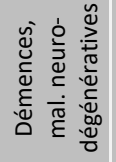 & 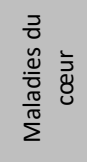 & 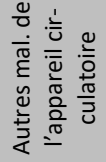 & 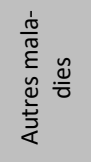 & 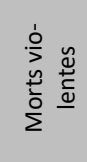 & 竞 \\
\hline \multicolumn{10}{|c|}{ Première période avant la canicule (1979-1992) } \\
\hline 90 & 0,099 & $-0,013$ & $-0,005$ & $-0,013$ & 0,028 & 0,052 & 0,025 & 0,007 & 0,018 \\
\hline 91 & 0,109 & $-0,010$ & $-0,011$ & $-0,007$ & 0,023 & 0,050 & 0,026 & 0,013 & 0,026 \\
\hline 92 & 0,060 & $-0,005$ & $-0,010$ & $-0,006$ & 0,016 & 0,035 & 0,012 & 0,003 & 0,015 \\
\hline 93 & 0,014 & $-0,013$ & $-0,008$ & $-0,007$ & 0,001 & 0,018 & 0,008 & 0,001 & 0,013 \\
\hline 94 & 0,010 & $-0,013$ & $-0,008$ & $-0,006$ & $-0,004$ & 0,024 & 0,004 & 0,004 & 0,009 \\
\hline 95 & 0,031 & $-0,003$ & $-0,007$ & $-0,004$ & 0,007 & 0,012 & 0,007 & 0,004 & 0,016 \\
\hline 96 & 0,005 & $-0,009$ & $-0,003$ & $-0,001$ & $-0,001$ & 0,009 & 0,004 & 0,002 & 0,004 \\
\hline 97 & $-0,003$ & $-0,003$ & $-0,002$ & 0,000 & $-0,003$ & 0,004 & $-0,003$ & 0,002 & 0,003 \\
\hline 98 & 0,009 & 0,000 & $-0,003$ & 0,000 & 0,003 & 0,006 & $-0,001$ & 0,000 & 0,005 \\
\hline 99 & $-0,002$ & $-0,002$ & $-0,003$ & 0,000 & $-0,001$ & 0,001 & 0,000 & 0,000 & 0,003 \\
\hline 100 & 0,007 & 0,000 & 0,002 & 0,000 & $-0,001$ & 0,003 & 0,002 & 0,001 & 0,001 \\
\hline 101 et + & 0,000 & 0,000 & 0,000 & 0,000 & 0,000 & 0,000 & 0,000 & 0,000 & 0,000 \\
\hline Somme & 0,340 & $-0,071$ & $-0,060$ & $-0,045$ & 0,068 & 0,214 & 0,085 & 0,036 & 0,113 \\
\hline \multicolumn{10}{|c|}{ Seconde période avant la canicule (1992-2002) } \\
\hline 90 & 0,050 & 0,022 & $-0,005$ & $-0,023$ & 0,008 & 0,029 & 0,004 & 0,007 & 0,009 \\
\hline 91 & 0,034 & 0,023 & $-0,003$ & $-0,022$ & 0,000 & 0,027 & 0,003 & 0,000 & 0,008 \\
\hline 92 & 0,017 & 0,011 & $-0,006$ & $-0,014$ & 0,001 & 0,019 & $-0,003$ & 0,006 & 0,004 \\
\hline 93 & 0,015 & 0,019 & $-0,007$ & $-0,014$ & $-0,002$ & 0,015 & 0,001 & 0,000 & 0,004 \\
\hline 94 & 0,021 & 0,014 & $-0,001$ & $-0,009$ & 0,004 & 0,012 & $-0,003$ & 0,001 & 0,003 \\
\hline 95 & 0,006 & 0,007 & 0,002 & $-0,007$ & $-0,002$ & 0,007 & $-0,001$ & $-0,001$ & 0,002 \\
\hline 96 & 0,004 & 0,007 & $-0,003$ & $-0,006$ & $-0,005$ & 0,007 & $-0,001$ & $-0,001$ & 0,005 \\
\hline 97 & $-0,002$ & 0,002 & $-0,002$ & $-0,003$ & $-0,006$ & 0,003 & 0,002 & 0,000 & 0,002 \\
\hline 98 & $-0,004$ & $-0,001$ & 0,000 & $-0,003$ & $-0,003$ & 0,002 & $-0,002$ & $-0,001$ & 0,002 \\
\hline 99 & 0,001 & 0,001 & $-0,001$ & $-0,002$ & 0,000 & 0,002 & 0,001 & 0,000 & 0,000 \\
\hline 100 & $-0,001$ & 0,001 & $-0,001$ & 0,000 & $-0,001$ & $-0,001$ & $-0,002$ & 0,000 & 0,002 \\
\hline 101 et + & 0,000 & 0,000 & 0,000 & 0,000 & 0,000 & 0,000 & 0,000 & 0,000 & 0,000 \\
\hline Somme & 0,140 & 0,105 & $-0,028$ & $-0,105$ & $-0,004$ & 0,123 & $-0,002$ & 0,011 & 0,040 \\
\hline \multicolumn{10}{|c|}{ Période après la canicule (2005-2015) } \\
\hline 90 & 0,094 & 0,009 & 0,017 & $-0,012$ & 0,046 & 0,017 & 0,012 & 0,000 & 0,006 \\
\hline 91 & 0,067 & 0,010 & 0,014 & $-0,015$ & 0,029 & 0,021 & 0,004 & 0,000 & 0,005 \\
\hline 92 & 0,041 & 0,008 & 0,002 & $-0,012$ & 0,021 & 0,015 & 0,003 & 0,000 & 0,004 \\
\hline 93 & 0,037 & 0,001 & 0,002 & $-0,008$ & 0,023 & 0,010 & 0,005 & $-0,002$ & 0,006 \\
\hline 94 & 0,030 & 0,005 & 0,006 & $-0,008$ & 0,013 & 0,009 & 0,003 & $-0,002$ & 0,004 \\
\hline 95 & 0,015 & 0,003 & 0,003 & $-0,003$ & 0,005 & 0,005 & 0,002 & 0,000 & 0,002 \\
\hline 96 & 0,012 & 0,002 & 0,002 & $-0,005$ & 0,006 & 0,002 & 0,003 & 0,000 & 0,002 \\
\hline 97 & 0,005 & 0,002 & 0,001 & $-0,001$ & 0,001 & 0,002 & 0,000 & 0,000 & 0,001 \\
\hline 98 & 0,004 & 0,000 & 0,000 & $-0,001$ & 0,001 & 0,001 & 0,001 & 0,000 & 0,001 \\
\hline 99 & 0,002 & 0,000 & 0,000 & 0,000 & 0,001 & 0,001 & 0,000 & 0,000 & 0,001 \\
\hline 100 & 0,001 & $-0,007$ & $-0,003$ & $-0,001$ & 0,000 & 0,006 & $-0,001$ & $-0,001$ & 0,008 \\
\hline 101 et + & 0,001 & 0,001 & $-0,001$ & $-0,005$ & 0,000 & 0,000 & $-0,002$ & 0,002 & 0,005 \\
\hline Somme & 0,310 & 0,034 & 0,042 & $-0,070$ & 0,144 & 0,089 & 0,030 & $-0,002$ & 0,044 \\
\hline
\end{tabular}


TABleau 6 Composantes par âge et cause de la différence d'espérance de vie à 90 ans entre les sexes en 1979, 2007 et 2015 (Années et millièmes d'années)

\begin{tabular}{|c|c|c|c|c|c|c|c|c|c|}
\hline \multirow[b]{2}{*}{ 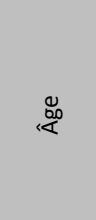 } & \multicolumn{9}{|c|}{ Grands groupes de causes } \\
\hline & 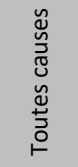 & 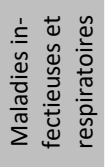 & 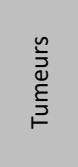 & 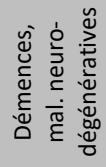 & $\begin{array}{l}\frac{3}{0} \\
\frac{y}{3} \\
\frac{0}{0} \\
\frac{\pi}{\pi} \\
\frac{\pi}{\pi}\end{array}$ & 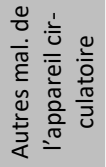 & 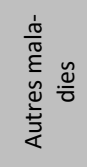 & 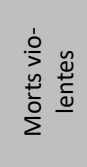 & 竞 \\
\hline \multicolumn{10}{|l|}{1979} \\
\hline 90 & 0,201 & 0,045 & 0,069 & $-0,003$ & 0,027 & 0,024 & 0,037 & 0,004 & $-0,002$ \\
\hline 91 & 0,155 & 0,033 & 0,034 & 0,001 & 0,026 & 0,023 & 0,027 & 0,007 & 0,005 \\
\hline 92 & 0,071 & 0,019 & 0,020 & $-0,001$ & 0,013 & 0,010 & 0,011 & 0,000 & $-0,001$ \\
\hline 93 & 0,066 & 0,022 & 0,020 & 0,000 & 0,010 & 0,007 & 0,015 & $-0,004$ & $-0,004$ \\
\hline 94 & 0,062 & 0,015 & 0,014 & $-0,001$ & 0,002 & 0,021 & 0,011 & 0,001 & $-0,001$ \\
\hline 95 & 0,028 & 0,010 & 0,004 & $-0,001$ & 0,002 & 0,002 & 0,007 & 0,001 & 0,002 \\
\hline 96 & 0,015 & 0,002 & 0,004 & 0,001 & 0,000 & 0,003 & 0,005 & 0,001 & $-0,001$ \\
\hline 97 & 0,009 & 0,007 & 0,007 & 0,002 & $-0,005$ & 0,002 & 0,003 & $-0,002$ & $-0,005$ \\
\hline 98 & 0,006 & 0,003 & 0,000 & 0,000 & 0,001 & 0,002 & 0,000 & $-0,001$ & 0,001 \\
\hline 99 & 0,003 & $-0,003$ & 0,002 & 0,001 & 0,000 & 0,003 & $-0,003$ & 0,001 & 0,002 \\
\hline 100 & 0,002 & 0,000 & 0,000 & 0,000 & 0,000 & 0,000 & 0,000 & 0,000 & 0,000 \\
\hline 101 et + & 0,001 & 0,000 & 0,000 & 0,000 & 0,000 & 0,000 & 0,001 & 0,000 & 0,000 \\
\hline Somme & 0,620 & 0,154 & 0,175 & 0,000 & 0,076 & 0,098 & 0,113 & 0,008 & $-0,004$ \\
\hline \multicolumn{10}{|l|}{2007} \\
\hline 90 & 0,243 & 0,046 & 0,084 & 0,003 & 0,064 & 0,012 & 0,020 & 0,018 & $-0,002$ \\
\hline 91 & 0,207 & 0,044 & 0,075 & 0,005 & 0,039 & 0,016 & 0,017 & 0,012 & $-0,001$ \\
\hline 92 & 0,147 & 0,035 & 0,054 & $-0,005$ & 0,035 & 0,004 & 0,012 & 0,010 & 0,001 \\
\hline 93 & 0,124 & 0,029 & 0,050 & $-0,006$ & 0,029 & 0,007 & 0,008 & 0,010 & $-0,002$ \\
\hline 94 & 0,086 & 0,021 & 0,030 & $-0,003$ & 0,021 & 0,006 & 0,007 & 0,005 & $-0,001$ \\
\hline 95 & 0,069 & 0,013 & 0,026 & $-0,006$ & 0,020 & 0,004 & 0,009 & 0,004 & 0,000 \\
\hline 96 & 0,051 & 0,016 & 0,015 & $-0,004$ & 0,010 & 0,002 & 0,008 & 0,003 & 0,001 \\
\hline 97 & 0,034 & 0,008 & 0,010 & $-0,001$ & 0,008 & 0,002 & 0,005 & 0,003 & 0,000 \\
\hline 98 & 0,022 & 0,008 & 0,007 & $-0,002$ & 0,004 & 0,002 & 0,002 & 0,003 & $-0,001$ \\
\hline 99 & 0,015 & 0,005 & 0,006 & $-0,003$ & 0,005 & 0,001 & 0,000 & 0,001 & 0,000 \\
\hline 100 & 0,009 & 0,003 & 0,002 & $-0,001$ & 0,002 & 0,001 & 0,001 & 0,001 & 0,000 \\
\hline 101 et + & 0,011 & 0,007 & 0,002 & $-0,004$ & 0,005 & $-0,001$ & 0,005 & 0,001 & $-0,004$ \\
\hline Somme & 1,020 & 0,235 & 0,360 & $-0,028$ & 0,244 & 0,055 & 0,094 & 0,069 & $-0,009$ \\
\hline \multicolumn{10}{|l|}{2015} \\
\hline 90 & 0,175 & 0,035 & 0,070 & $-0,006$ & 0,036 & 0,007 & 0,020 & 0,012 & 0,000 \\
\hline 91 & 0,159 & 0,029 & 0,052 & $-0,002$ & 0,041 & 0,006 & 0,019 & 0,014 & 0,000 \\
\hline 92 & 0,131 & 0,022 & 0,047 & $-0,002$ & 0,033 & 0,004 & 0,015 & 0,011 & 0,000 \\
\hline 93 & 0,102 & 0,023 & 0,043 & $-0,006$ & 0,023 & 0,000 & 0,012 & 0,008 & $-0,001$ \\
\hline 94 & 0,080 & 0,019 & 0,028 & $-0,006$ & 0,019 & 0,002 & 0,011 & 0,009 & $-0,002$ \\
\hline 95 & 0,058 & 0,015 & 0,020 & $-0,009$ & 0,012 & 0,001 & 0,012 & 0,006 & 0,000 \\
\hline 96 & 0,042 & 0,012 & 0,014 & $-0,005$ & 0,010 & 0,001 & 0,006 & 0,005 & $-0,001$ \\
\hline 97 & 0,030 & 0,009 & 0,008 & $-0,003$ & 0,007 & 0,001 & 0,006 & 0,002 & 0,000 \\
\hline 98 & 0,017 & 0,009 & 0,006 & $-0,003$ & 0,005 & $-0,001$ & 0,002 & 0,001 & $-0,001$ \\
\hline 99 & 0,011 & 0,005 & 0,004 & $-0,003$ & 0,003 & 0,000 & 0,000 & 0,003 & $-0,001$ \\
\hline 100 & 0,007 & 0,005 & 0,002 & $-0,003$ & 0,002 & $-0,001$ & 0,002 & 0,001 & $-0,001$ \\
\hline 101 et + & 0,007 & 0,004 & 0,002 & $-0,002$ & 0,001 & 0,000 & 0,002 & 0,001 & 0,000 \\
\hline Somme & 0,820 & 0,187 & 0,297 & $-0,049$ & 0,191 & 0,020 & 0,109 & 0,074 & $-0,009$ \\
\hline
\end{tabular}

